PRE-SHOT SIMULATIONS OF NEAR-FIELD AND FAR-FIELD GROUND MOTION FOR THE SOURCE PHYSICS EXPERIMENT (SPE) EXPLOSIONS AT THE CLIMAX STOCK, NEVADA NATIONAL SECURITY SITE: SPE3

R. J. Mellors, A. Pitarka, A. J. Rodgers, W. R. Walter, S. Ford, H. Xu, E. Matzel, S. Myers, N. A. Petersson, B. Sjogreen, T. Hauk, J. Wagoner, O. Vorobiev, L. Glenn, E. Herbold, T. Antoun, I. Lomov

July 20, 2012 
This document was prepared as an account of work sponsored by an agency of the United States government. Neither the United States government nor Lawrence Livermore National Security, LLC, nor any of their employees makes any warranty, expressed or implied, or assumes any legal liability or responsibility for the accuracy, completeness, or usefulness of any information, apparatus, product, or process disclosed, or represents that its use would not infringe privately owned rights. Reference herein to any specific commercial product, process, or service by trade name, trademark, manufacturer, or otherwise does not necessarily constitute or imply its endorsement, recommendation, or favoring by the United States government or Lawrence Livermore National Security, LLC. The views and opinions of authors expressed herein do not necessarily state or reflect those of the United States government or Lawrence Livermore National Security, LLC, and shall not be used for advertising or product endorsement purposes.

This work performed under the auspices of the U.S. Department of Energy by Lawrence Livermore National Laboratory under Contract DE-AC52-07NA27344. 


\section{PRE-SHOT SIMULATIONS OF \\ NEAR-FIELD AND FAR-FIELD GROUND \\ MOTION FOR THE SOURCE PHYSICS EXPERIMENT (SPE) EXPLOSIONS AT THE CLIMAX STOCK, NEVADA NATIONAL SECURITY SITE: SPE3}

LAWRENCE LIVERMORE NATIONAL LABORATORY

July 17,2012

LLNL-TR-XXXX

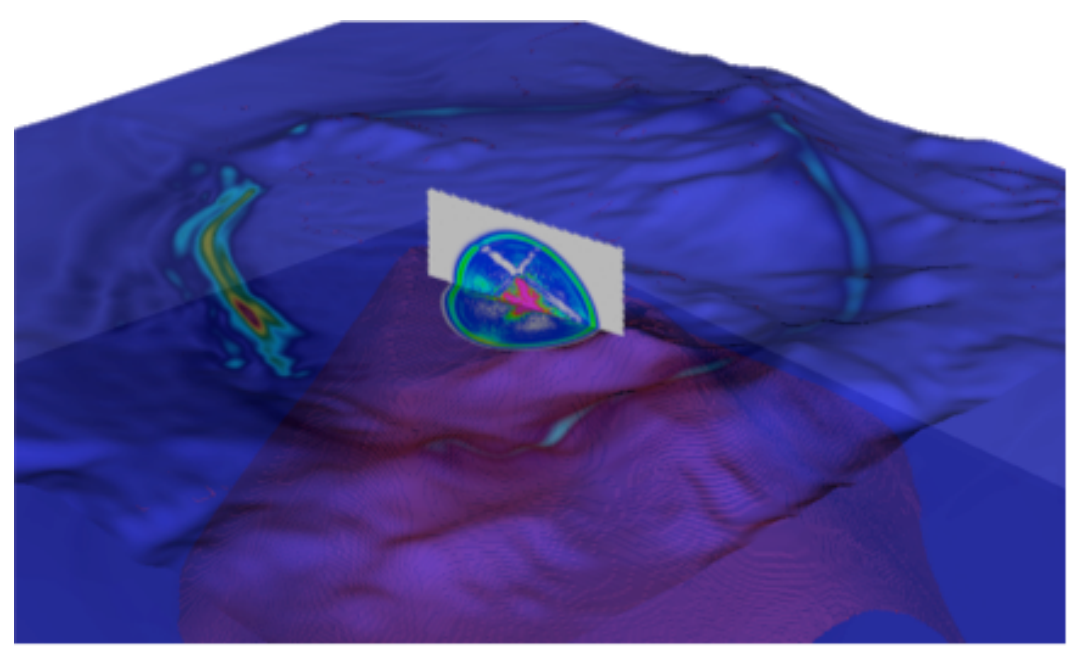




\section{TABLE OF CONTENTS}

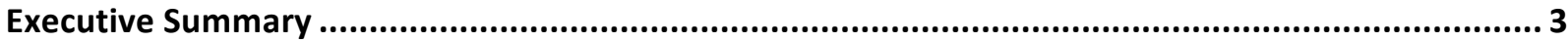

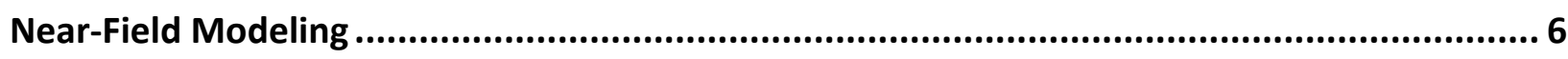

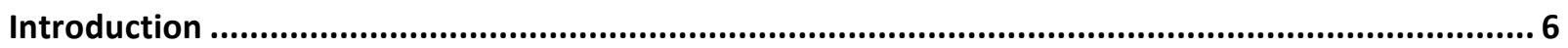

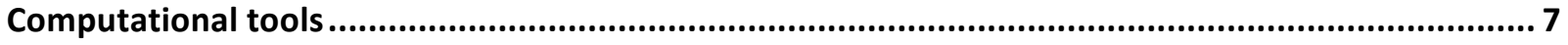

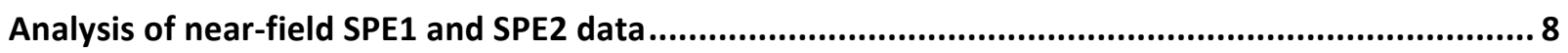

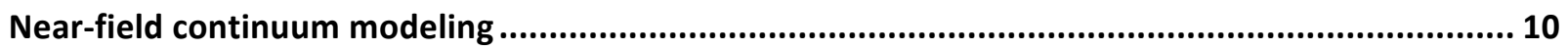

Granite model for small samples and its extension to the large scale........................................ 12

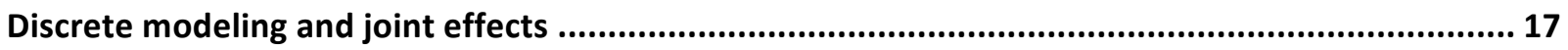

Continuum simulations of SPE3 event using ensemble model.................................................. 23

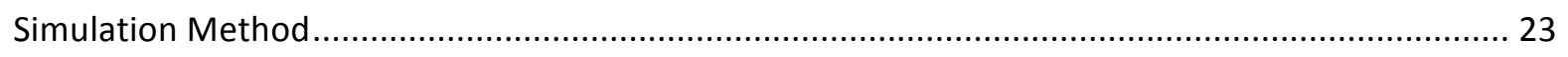

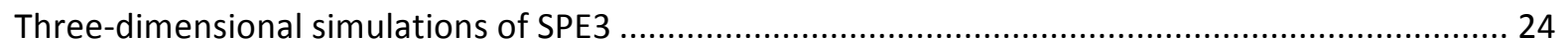

Comparison between wavefields across the fault plane ................................................................ 30

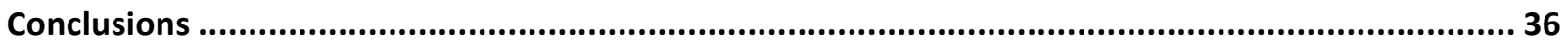

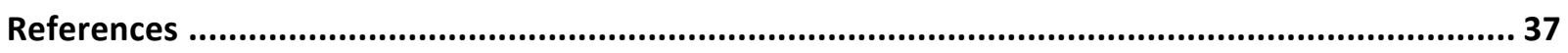

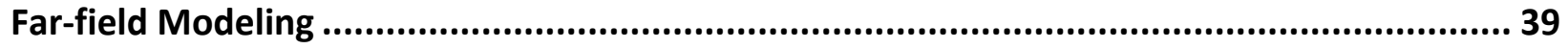

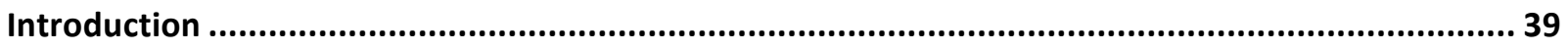

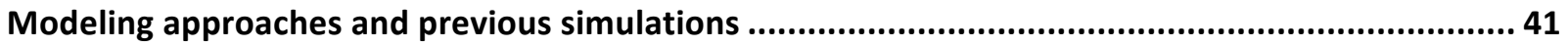

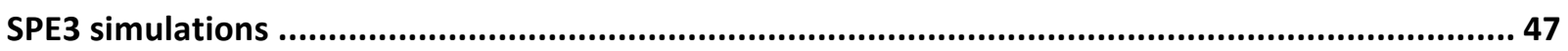

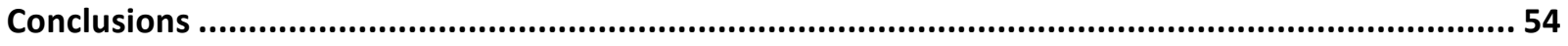

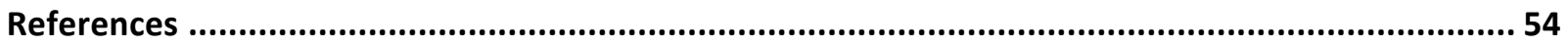




\section{EXECUTIVE SUMMARY}

The Source Physics Experiment (SPE) is designed to improve ground motion prediction capabilities for underground nuclear testing through the development of new physics-based models together with validation via new chemical explosion data. The SPE shots consist of different size and depth chemical explosions in a granite borehole at the Climax Stock on the Nevada National Security Site (NNSS). The third shot in the series (SPE3) will be the same size (1000 kg TNT equivalent) and depth (45 m canister centroid) as the previous shot (SPE2). The main purpose of the SPE3 is to investigate the effects of the damage zone caused by the SPE2 chemical explosion on the SPE3 explosion, keeping all other parameters (e.g. size, depth, explosive type) the same. This SPE3 pre-shot report addresses progress made in simulation of the expected wavefield in the both the near- and the far-field. In this report we consider the far-field to be where seismic wave motion can be characterized by elastic or visco-elastic approximations, in contrast to the near-field where other, often non-linear effects, play a major role. The current simulations build on previous work from the SPE1 and SPE2 shots. The simulations are intended to highlight differences between SPE2 and SPE3 using a variety to approximations intended to mimic the expected effect of the damage zone.

The simplest physics of damage zone effects suggest changes in the source region material properties (density, velocity), and in the yield strength (coupling) of the rock, should both impact the far-field signal. We can illustrate this in a simplistic way for P-waves in Figure A using the empirical Mueller-Muphy (1971) far-field explosion model (MM). First if the SPE2 explosion-induced damage reduces velocities and densities without affecting the yield strength or coupling, then by energy conservation the amplitudes for SPE 3 would increase as shown in the top of Figure A. Similarly if the explosion induced damage increased density (say by compacting pore space) the amplitude would decrease. This impedance effect, where the product of the density, wave-velocity, and ground-velocity squared is conserved, is well known. The second and harder effect to quantify, are changes in the energy coupling or yield strength. In the simple MM model this is represented by the coupling factor between cavity radius and yield. If we assume the granite was slightly weakened by SPE2 such that the cavity radius increases slightly then the overall amplitudes of SPE3 decrease relative to SPE2 as shown in the bottom of Figure A. In reality both effects, may exist due to the damage zone, and furthermore we know they are interrelated in complex ways. For S-waves we expect even more complex effects due to motion on joints and faults. For this reason we are developing physics-based near field simulation models coupled to far-field propagation codes to predict the overall effects of changes in the source emplacement region on observed signals.

Near-field simulations have been performed using the Eulerian code (GEODYN). An ensemble model, calibrated to match records from past large-scale nuclear events is used. Both 2D axisymmetric (no faults) and fully three-dimensional GEODYN calculations (with /without faults) were conducted in which the damage zones sustained by SPE2 are retained as the initial condition for the SPE3 calculations. The results indicate that the waveform peak amplitudes are slightly decayed since the ensemble model includes no softening mechanism. The pulse widths are reduced, indicating the energy is dissipated more by the damage zone as expected. The dominant motions calculated in all the scenarios are spherically symmetric and non-spherical components are small as seen for the SPE2 calculations. The arrival time of the SPE3 waveform at the boreholes is shorter than SPE2 due to compaction during the previous explosion. As shown in the SPE 1 and 2 observations shear waves are generated, indicating the potential contributions from the induced motion on distributed joints. The effects of joints are evaluated using the 
Lagrangian hydrocode (GEODYN-L), which was benchmarked against the GEODYN calculations for the ensemble model. A new granite model calibrated for samples from the SPE site is used. The new model includes effects of poroelasticity and water saturation and may be scaled allowing application to both small and large scales. Parametric tests were performed to evaluate the effect of joints within the granite and the results demonstrate that in situ joints introduce anisotropy and significant tangential motions.
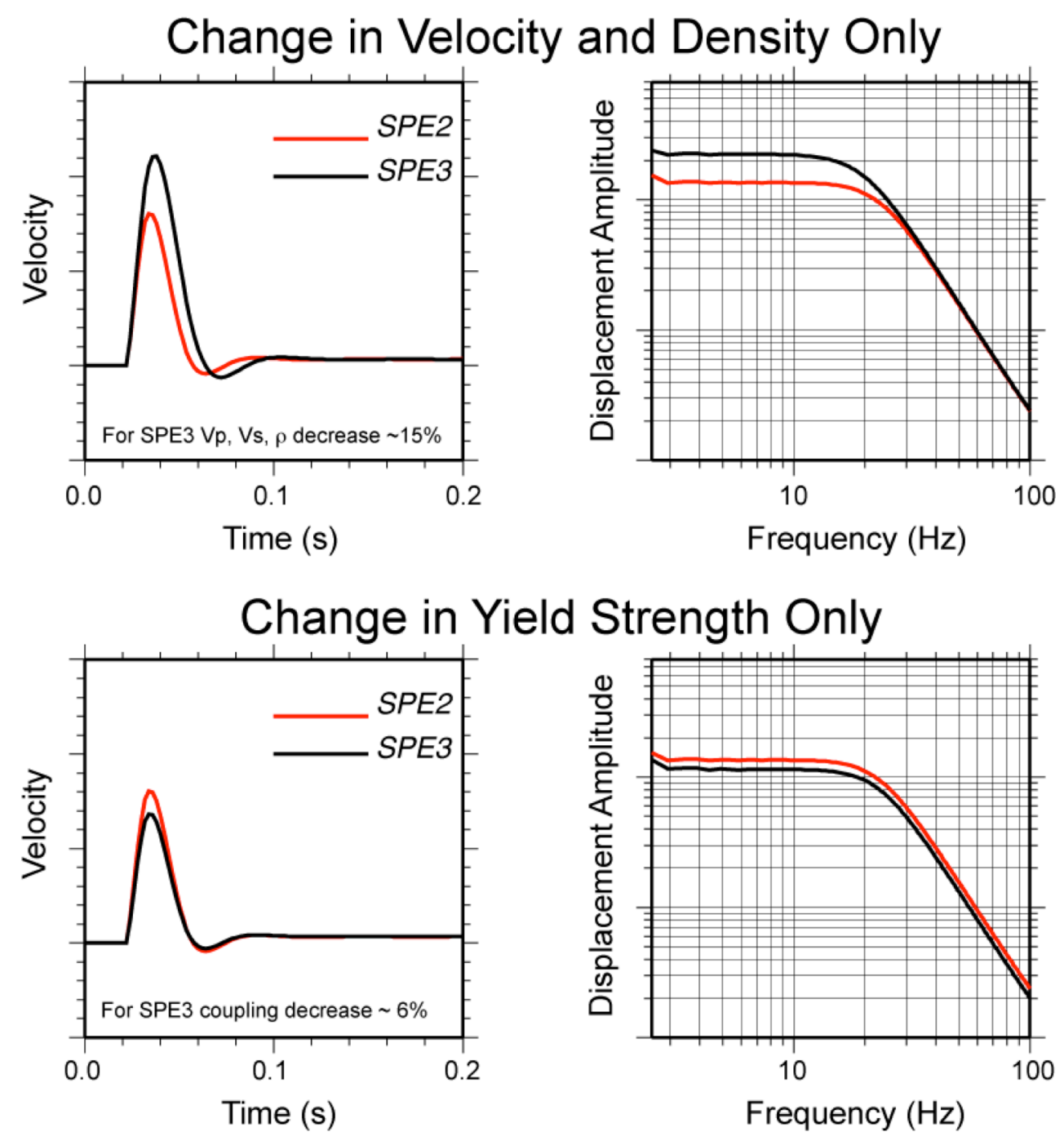

Fig. A. Highly simplified far-field predictions of P-wave changes between SPE3 and SPE2 in the time (left-hand-side) and frequency (right-hand side) domain due to changes in velocity-density (top) and yield strength (bottom).

Far-field simulations using both numerical and semi-empirical methods have been conducted for the upcoming SPE3 shot. These simulations include: 1) changes to both the algorithms and the velocity 
models to improve the data fit based on previous shots and 2) modifications to simulate the effect of a damage zone. Earth model changes include the addition of a low-velocity layer near the granite surface and the addition of random heterogeneities in the $3 \mathrm{D}$ velocity model to increase scattering. The 3D finite-difference simulations tested two different sources: a source time function representing the Mueller-Murphy sources shown in Figure A, and a direct 3D coupling with the near-field source models generated by GEODYN. Initial results with the direct 3D coupling are promising but are undergoing validation. The semi-empirical models scale the SPE3 waveforms from SPE2 to compensate for the expected change in the source spectrum caused by the near-source damage zone. In general the far-field simulations predict only relatively small changes in amplitude and frequency for SPE 3 compared to SPE2 beyond $100 \mathrm{~m}$. 


\title{
NEAR-FIELD MODELING
}

\author{
OLEG VOROBIEV, HEMING XU, LEW GLENN, ERIC HERBOLD, TARABAY ANTOUN AND \\ ILYA LOMOV
}

\section{INTRODUCTION}

Motion along joints and fractures in the rock has been proposed as one of the sources of near-source shear wave generation, and demonstrating the validity of this hypothesis is a focal scientific objective of the source physics experimental campaign in the Climax Stock granitic outcrop. A modeling effort has been undertaken by LLNL to complement the experimental campaign, and over the long term provides a validated computation capability for the nuclear explosion monitoring community. Our approach involves performing the near-field nonlinear modeling with hydrodynamic codes (e.g., GEODYN, GEODYN-L), and the far-field seismic propagation with an elastic wave propagation code (e.g., WPP). The codes will be coupled together to provide a comprehensive source-to-sensor modeling capability. Our technical approach involves pre-test predictions of each of the SPE experiments using our state of the art modeling capabilities, followed by code improvements to alleviate deficiencies identified in the pre-test predictions. This iterative development cycle wherein simulations are used to guide experimental design and the data from the experiment used to improve the models is the most effective approach to enable a transition from the descriptive phenomenological models in current use to the predictive, hybrid physics models needed for a science-based modeling capability for nuclear explosion monitoring.

The objective of this report is to describe the preliminary predictions of the $3^{\text {rd }}$ SPE shot in the Climax Stock (Schock et al, 1973), a 2576lb HE at a depth of 150ft (SPE3). That is the same location and yield of the SPE2 in order to quantify the effect of the damage caused by SPE2 on the outgoing wavefields. The simulations are performed using the LLNL ensemble granite as for SPE1 and SPE2 (Antoun et al 2001; Antoun et al. 2011) for base rock model and weak porous granite model for the top $20 \mathrm{~m}$ layer. The 2D axisymmetric (performed without the top layer) and full 3D simulations are performed and the two faults are incorporated into the calculations in 3D as for SPE1 and SPE2. The SPE3 predictions are compared with the SPE2 results and the wavefield variations across the two faults at the shot level are also presented below. Note Hole \#1A is the replacement hole of \#1 and the results are similar at those two holes so the simulations at \#1A are not presented below although they are both calculated. The plane view of the borehole locations is shown in Figure 1a. 


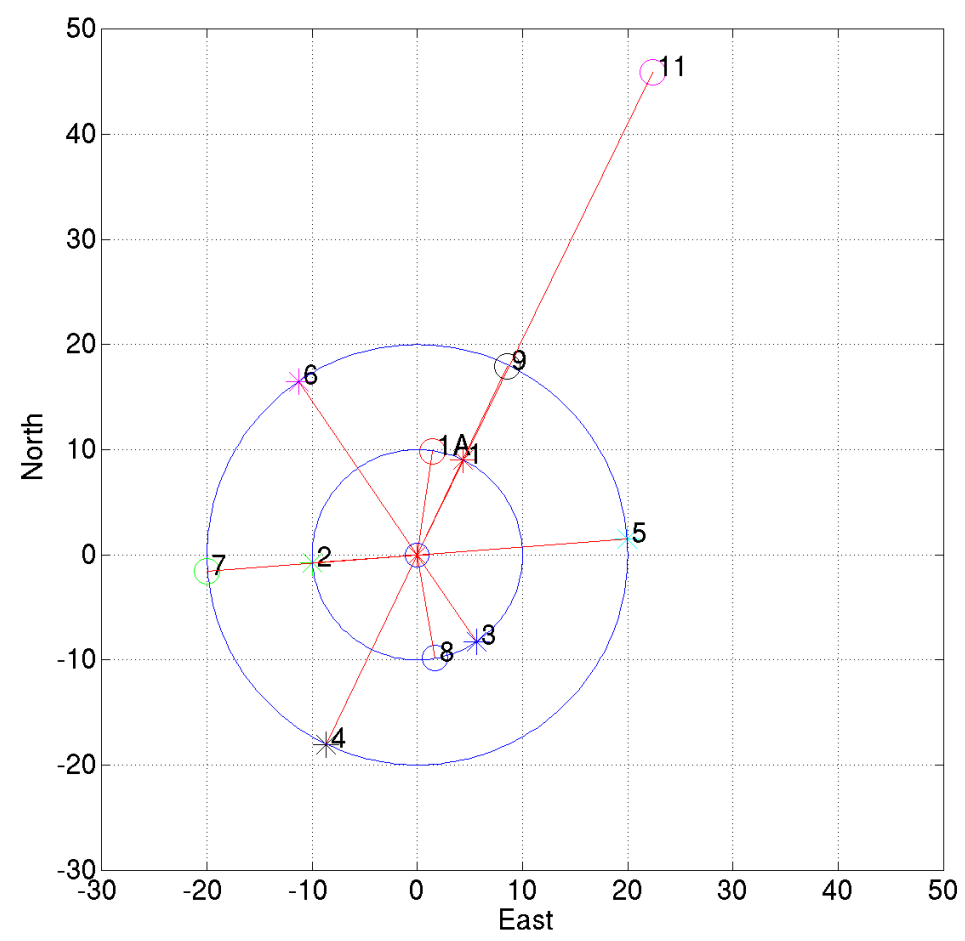

Figure 1. Plane view of the borehole locations for SPE3.

\section{COMPUTATIONAL TOOLS}

GEODYN: Most of the continuum simulations described here were performed using GEODYN-a massively parallel three-dimensional Eulerian code with adaptive mesh refinement capabilities (Lomov and Liu, 2005). The constitutive model used in the simulations is the LLNL 'Ensemble' granite model. This model is nonlinear, thermodynamically consistent, and properly invariant under superposed rigid body motions (Rubin et al. 2000). The model assumes the material is isotropic and applies the mathematical structure of plasticity theory to capture the basic features of the mechanical response of geological materials, including the effects of bulking, yielding, material damage, and porous compaction on the material response. This model has been shown to be in good agreement with the static data of Schock et al. (1973), as well as explosion data in granite [Antoun et al. (2001)]. One of the shortcomings of this model is that it does not account for directional effects associated with wave propagation. Alleviating this shortcoming is one of the main objectives of the near-field modeling effort.

GEODYN-L: Discrete simulations were performed using a Lagrangian massively parallel three-dimensional code, GEODYN-L (Vorobiev, 2011). This code was designed to model wave propagation through heavily jointed rock masses. Joints are treated using an advanced contact algorithm 
with history variables. In the simulations, the geological medium was represented as an assembly of intact rock blocks separated by joints. Two material models were used in GEODYN-L. The first, the LLNL 'Ensemble' granite model, was used to verify that both codes give the same results for the SPE1 problem modeled as a spherical explosion. The second, an intact model [Vorobiev, 2008] was built to match available lab data for the granite samples from the site. Figure 5 shows an unconfined compressive strength test simulated with that model. The intact granite model was used to model response within each block of the rock mass. The net response depended on both intact material and joint models.

\section{ANALYSIS OF NEAR-FIELD SPE1 AND SPE2 DATA}

We have analyzed the near-field measurement data from two consequent experiments (SPE1 and SPE2). Because of uncertainty in possible gauge rotation used in the experiments we will focus in our analysis on quantities invariant to the orientation of the gauges such as total velocity magnitude and vertical velocities at depths of $150 \mathrm{ft}$ and $180 \mathrm{ft}$.

Figure 2 shows the peak total velocity amplitudes obtained from the borehole records for SPE1 (triangles) and SPE2 (circles). The locations of the boreholes are given in (Antoun et al., 2011). The peak amplitudes from the historical nuclear explosions at Degelen, HardHat, Hoggar, PileDriver and Shoal are displayed with the small black symbols. It is seen that the relationships between the peak velocities for SPE1 and SPE2 and scaled distance are quite consistent in this scale range and both are also in agreement with those measurements from the hard rock nuclear explosions (small black symbols). The line is the regression curve for nuclear explosions in granite model for reference.

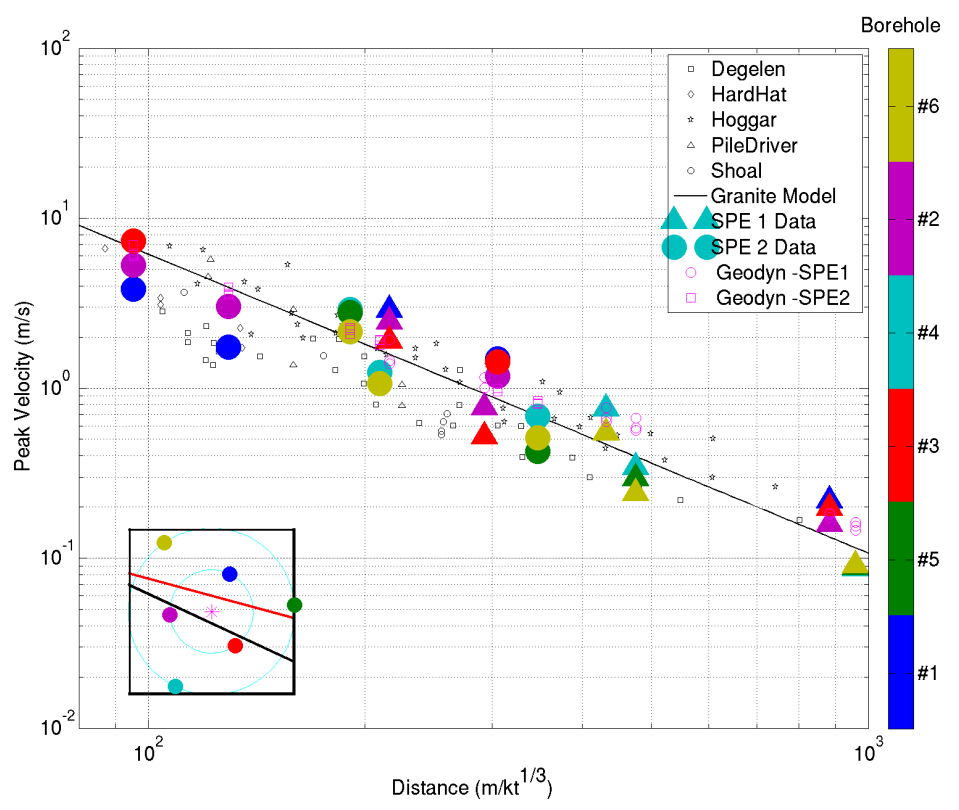


Figure 2. Peak total velocity amplitudes versus scaled distance for SPE 1 (triangle) and SPE 2 (circle). The peak velocities from the hardrock nuclear explosions are indicated by the small symbols. The continuum modeling results are displayed for SPE 1 (small red circle) and SPE 2(small red square). The line is the regression model for the nuclear explosions in granite. The inset shows the location of the 6 borehole locations and the locations of the two faults at 150ft.
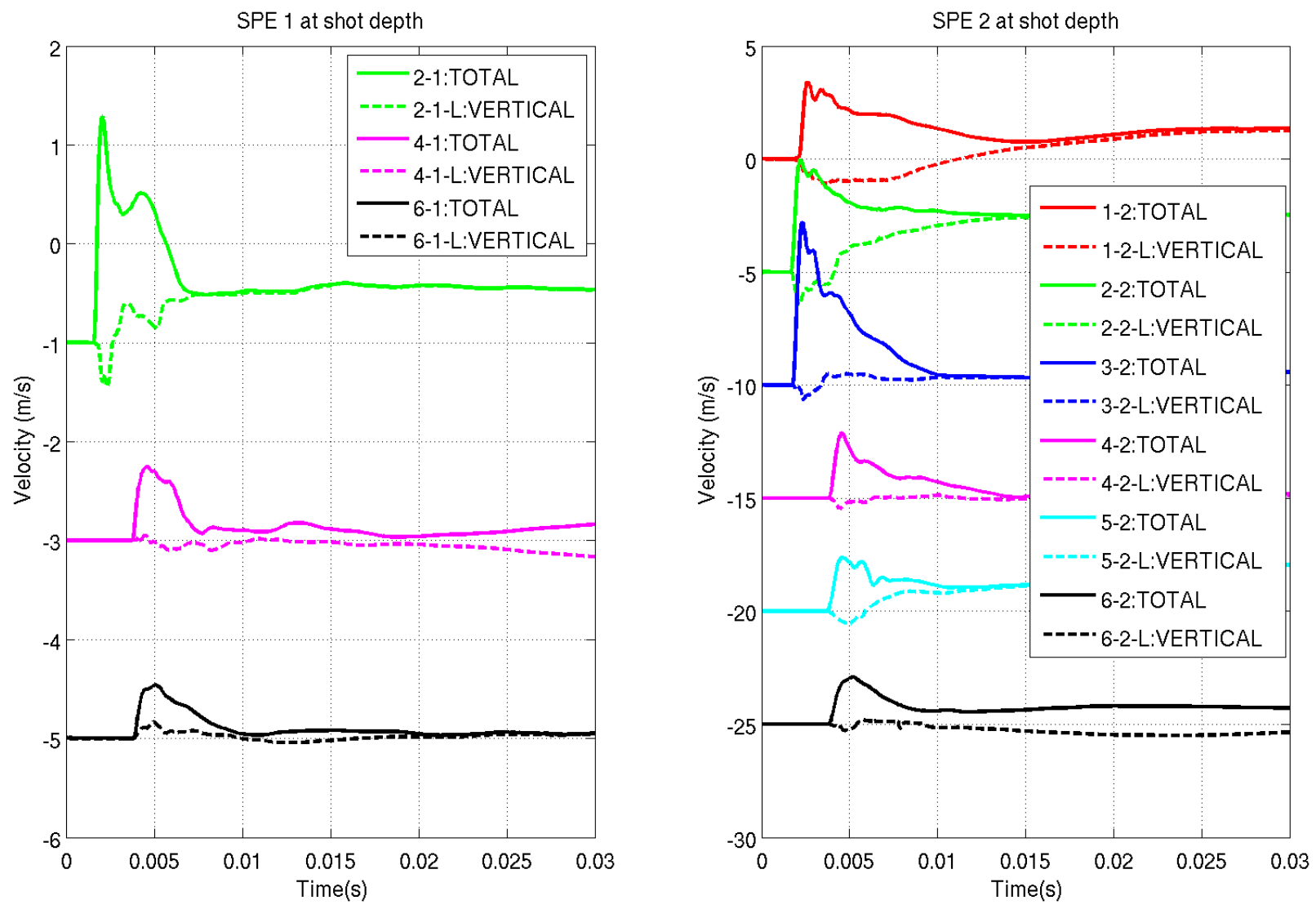

Figure 3. Vertical motions (dashed lines) at the shot levels of SPE1 (left) and SPE2 (right) in comparison with the total amplitudes at the gages.

Although the peak velocity magnitudes for SPE 1 and SPE 2 decay at a similar rate as for the historical explosions and show comparable azimuthal variations, detailed studies on the borehole records show that the SPE 1 and SPE 2 explosions are not purely spherical. 

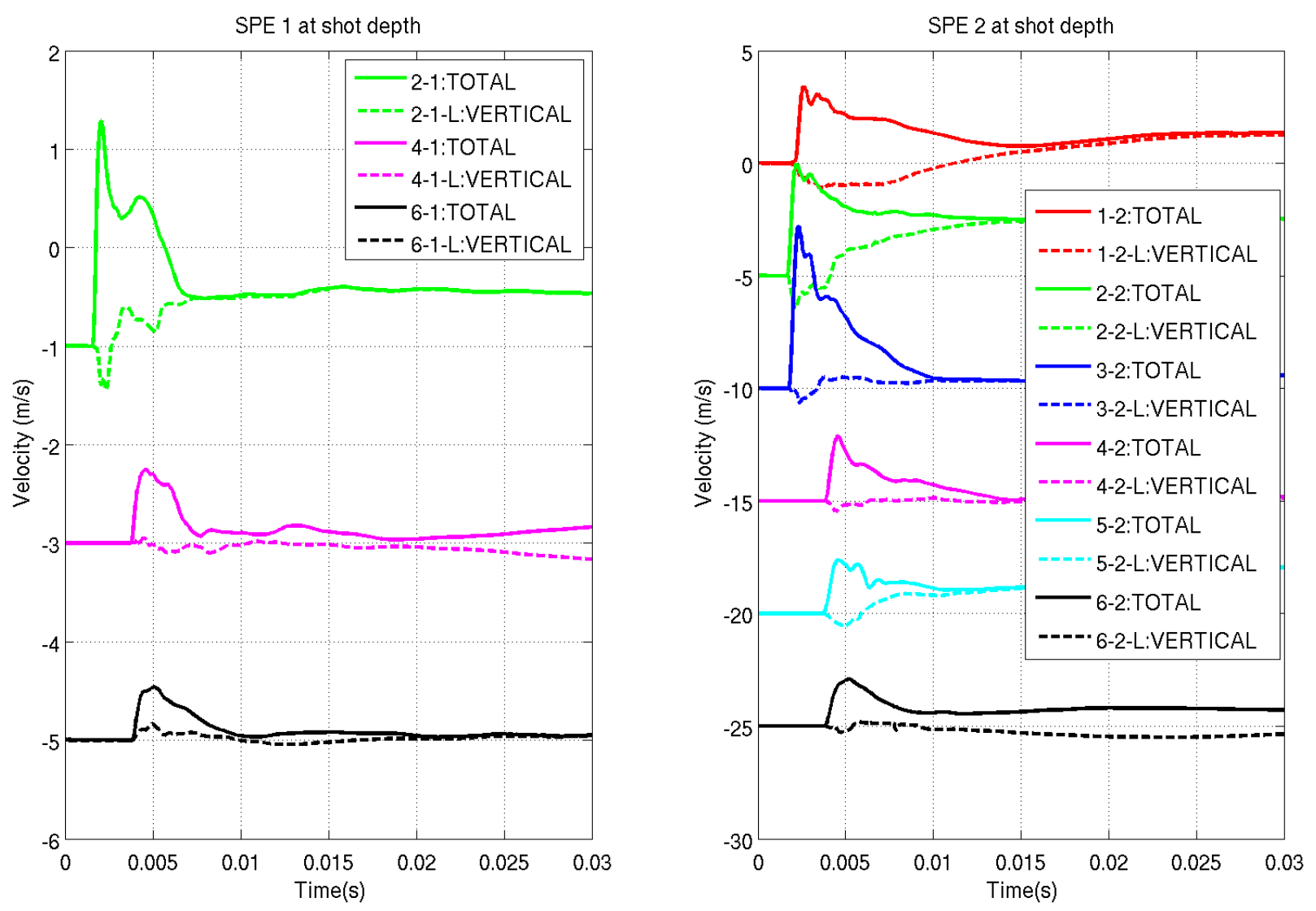

Figure 3 shows the comparison of the vertical motion with the total velocities for SPE1 (left) and for SPE2 (right). The solid lines represent the total velocity amplitude and the dashed lines display vertical velocities (upward positive). There are only 3 gauges with complete 3-component records for SPE 1 (left) at the shot depth while 6 gauges for SPE 2 (right). The gage locations are shown in the inset of Figure 2. It is evident at the borehole 2 (green, $10 \mathrm{~m}$ distance), the vertical motions are not negligible relative to the total amplitudes for both SPE 1 and SPE2 and also clear in borehole 1 and 3 records for SPE 2 at the shot depth. At a larger distance (boreholes 4-6, 20m) the vertical motion decays with distance as expected but the relative amplitudes are still not negligible. All these vertical motion observations confirm that the wave fields generated by SPE 1 and SPE 2 are not spherical, i.e., the shear waves are certainly generated in the vicinity of the source region and might be related to the onsite heterogeneities such as fractures, joints and faults as observed in the borehole logs.

\section{NEAR-FIELD CONTINUUM MODELING}

An Eulerian hydrocode, GEODYN (Antoun et al, 2001), and a Lagrangian hydrocode, GEODYN-L (Vorobiev, 2011) were used to calculate spherical wave propagation both in 2D and 3D. The (ensemble) constitutive model employed was derived by calibrating to match peak velocities and displacements for a 
number of underground nuclear explosions of different magnitude in granitic rock conducted both in the US and abroad as seen in Figure 2 (Antoun et al, 2001).

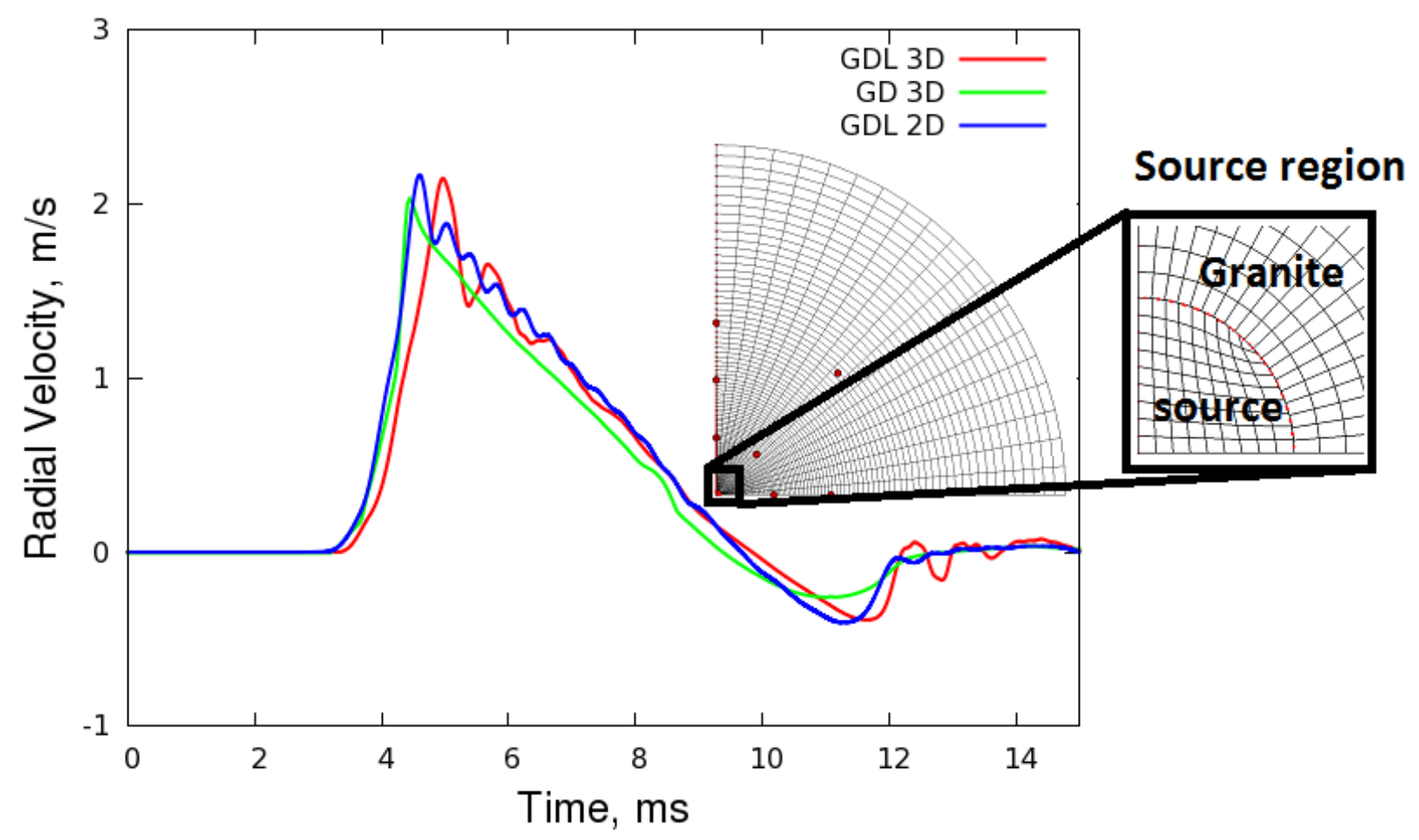

Figure 4. Comparison between $3 D$ GEODYN calculations, $2 D$ axisymmetric GEODYN-L and $3 D$ GEODYN-L calculations for a spherical explosion in granite. The ensemble model was used in both codes and the yield corresponded to SPE2 event.

Figure 4 shows a comparison between the Lagrangian and Eulerian calculations for SPE 2 using the same ensemble model where the radial velocity history at $20 \mathrm{~m}$ range is plotted. The differences between these results are within a few percents and are explained by the mesh resolution around the source (for example, longer rise time for the Lagrangian results can be explained by transitioning between the meshes used for the spherical source and the region occupied by the granite) and artificial viscosity (extra oscillations after the shock observed in both 2D and 3D Lagrangian calculations).

The ensemble model is an isotropic plasticity model designed for large scale simulations. It does not use small scale test data for calibration and assumes that any joints in the granite formation are distributed uniformly resulting in an isotropic material response. Since the model is isotropic, it cannot explain the observed variability in the data, deviations from sphericity and the generation of significant shear (transverse) waves from explosions. The wave motion data from SPE on the other hand exhibit significant non-radial motions, even at the $10 \mathrm{~m}$ range, the closest gage location to the explosive source. Logging data from the instrumentation bore holes, as well as from the SPE charge emplacement hole indicate that the 
granite is highly fractured, with an average joint spacing of about $1 \mathrm{ft}$. In addition, the logging data shows that the joints are not randomly orientated; instead four joint sets have been identified; each of which exhibiting a distinct orientation with relatively little variability. These features are conveniently incorporated into the discrete models in GEODYN-L as follows.

\section{GRANITE MODEL FOR SMALL SAMPLES AND ITS EXTENSION TO THE LARGE SCALE}

The model developed in (Vorobiev et al 2007; Vorobiev, 2008) was used to describe the constitutive behavior of the intact granite. It describes the effects of bulking, porous compaction, shear enhanced compaction, and pressure dependent yield on the material response. The yield strength function is chosen in the form

$$
\begin{aligned}
& Y=Y_{H B} F(\beta)\left[\delta+(1-\delta)\left(\frac{P_{c}-\max \left(P_{0}, P^{*}\right)}{P_{c}-P_{0}}\right)^{r}\right] R(\dot{\varepsilon}), Y_{H B}=Y_{c}\left[\sqrt{s+\frac{m^{2}}{36}+\frac{m P^{*}}{Y_{c}}}-\frac{m}{6}\right], \\
& 0<\delta=\frac{\varepsilon_{p}}{\varepsilon_{p 0}+\varepsilon_{p}}<1, r>1, P^{*}=P / R(\dot{\varepsilon})
\end{aligned}
$$

where $\beta$ is the Lode angle, $Y_{c}$ is the unconfined compressive strength, $\delta$ is a function of plastic strain describing material hardening, $P_{c}$ and $P_{0}$ are history dependent variables describing a cap surface that governs porous compaction and $Y_{H B}$ is the ultimate compressive strength. The coefficients $s$ and $m$ depend on the quality of the rock as expressed by the Geological Strength Index (GSI), a rock quality index widely used by the geotechnical engineering community:

$$
s=\exp \left(\frac{G S I-100}{9}\right), m=m_{i} \exp \left(\frac{G S I-100}{28}\right)
$$

For the intact material, $s=1$ and the value $m_{i}$ can be found from static laboratory tests on intact samples. 


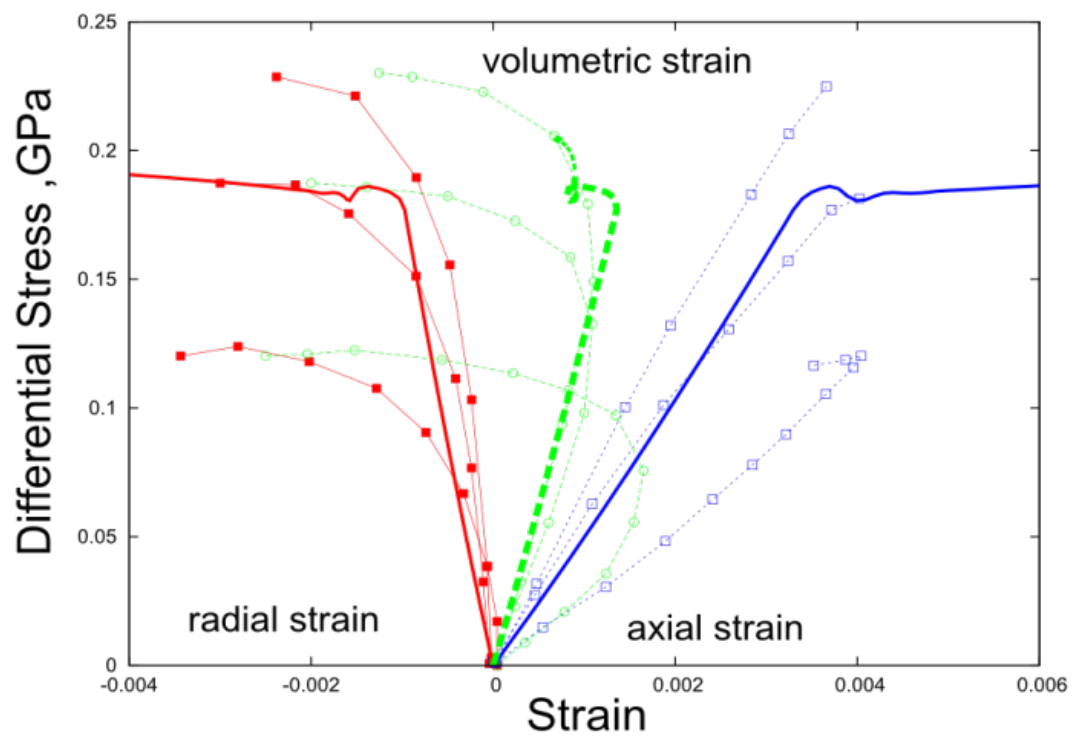

Figure 5. Comparison of model fits to unconfined compressive strength test for intact granite samples from the SPE test bed. Bold lines are simulation results, and thinner lines with markers are experimental data (Broome, 2011).

The model was calibrated to match intact granite data from the literature, as well as the unconfined compressive tests performed on the granite samples collected at the SPE1 site (see Figure 5). The model can be scaled to field scale by changing the GSI index, introducing extra porosity associated with the joints and modifying the porous compaction law. Figure 7 shows, that while the small scale model does not reproduce the wave attenuation data for large scale explosions, such as SPE1, it can be tuned to match the data by changing the GSI index, which describes the quality of rock mass. In this case, a GSI=60 was needed to match the measured displacement, which appropriately corresponds to a blocky rock with fair-to-poor surface conditions. In the mesoscale simulations described below, we used the model for intact granite, because the joints are included explicitly in the simulations. The model account for poroelasticity in porous rocks, which can be attributed to the elastic closing of the microcracks. Details of the model describing the poroelasticity implementation can be found in (Vorobiev, 2008).The initial bulk modulus is related to the reference porosity, $\Phi$, the poroelasticity parameters, a, and the solid bulk modulus $K_{s}$, as

$$
K_{0}=K_{s}(1-\Phi)(1-a)^{2}, \quad K_{s}=-J_{s} \frac{\partial P_{s}}{\partial J_{s}}
$$


It is known from experimental observations that the joint normal closure is a non-linear function of the applied normal stress resembling a hyperbola (Bandis et al., 1983). Therefore the normal modulus of the joint, ${ }^{E_{j}}$, can be expressed as

$$
E_{j}=E_{j 0} \frac{u_{0}^{2}}{\left(u_{0}-u_{\max }\right)^{2}}
$$

where $u_{0}$ is the aperture of the joint and $u_{\max }$ is the maximum joint closure for all times. Joint special density and their initial elastic normal and shear moduli, $E_{j 0}$ and ${ }^{G_{j 0}}$ will define how much the bulk modulus of jointed rock material should be reduced, and thus will define the poroelasticity parameter $a$. In continuum approach we assume that the joints are distributed uniformly and material remains isotropic. Fossum (1985) derived the following formula for the average bulk modulus in a randomly jointed elastic media with compliant joints

$$
K_{e f f}=\frac{E_{s}}{9}\left[\frac{3(1+v) s E_{j} / a+2 E_{s}}{(1+v)(1-2 v) s E_{j} / a+(1-v) E_{s}}\right] \text {, }
$$

where $s$ is an average joint spacing, $E_{s}$, is the Youngs modulus and $\mathrm{n}$ is the Poisson ratio assuming a linear behaviour of joints. Thus the value of poroelasticity parameter,a, can be estimated if joint density as well as basic elastic properties are known. 


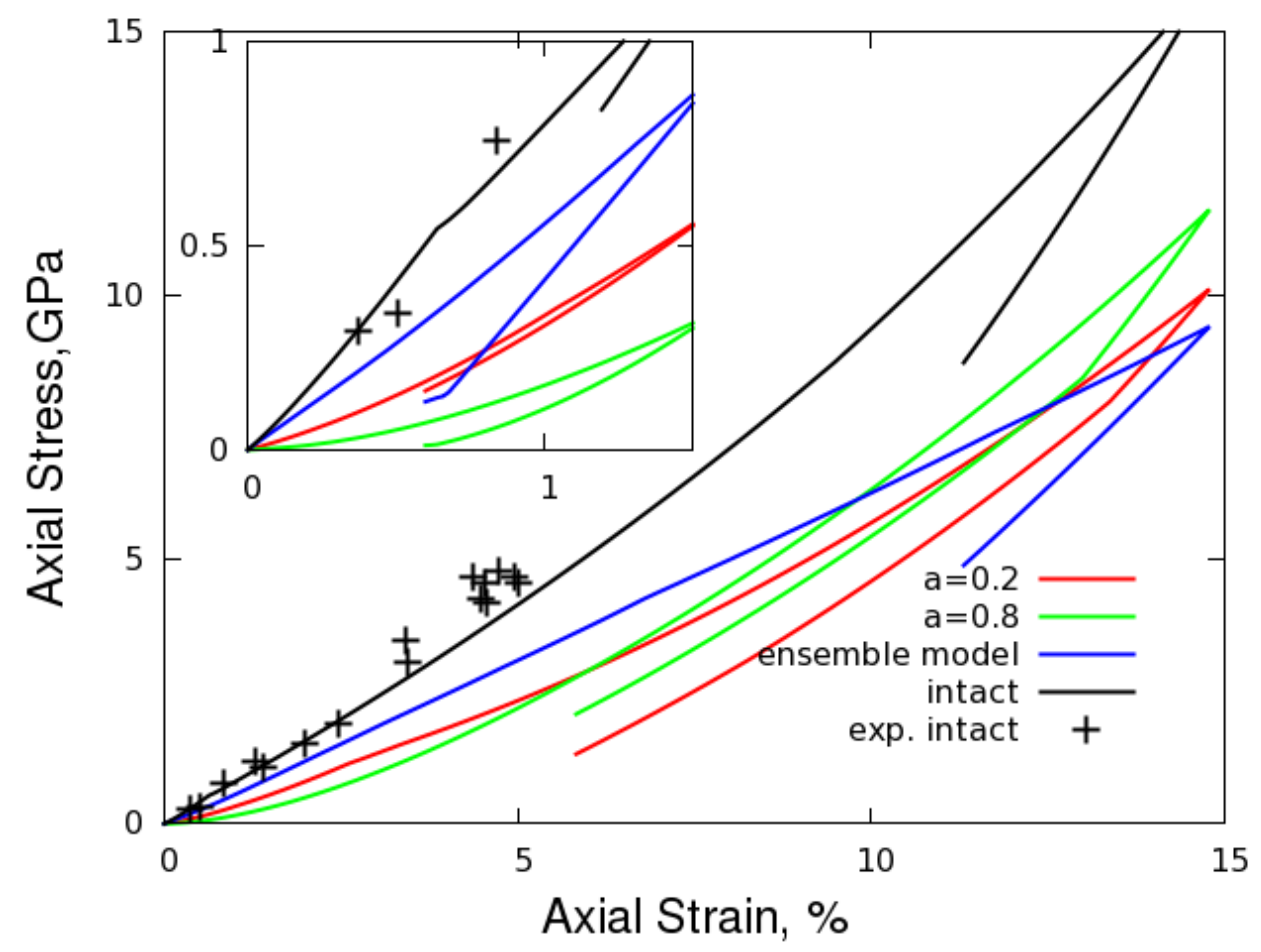

Figure 6. Effect of the poroelasticity parameter, a on the stress-strain response for the insitu model. Intact model results $(G S I=100)$ as well as the experimental data for granite samples from the area are shown with the marker points

Figure 6 shows response of various granite materials in uniaxial strain compression. The intact model was calibrated using lab scale measurements for small granite sample from the area (shown with points). To represent increased poroelasticity caused by the joints parameter, a, was increased to 0.2 , also GSI scaling was applied to reduce the yield surface as described above. For the top layer, we assumed that the joint density was higher and therefore increased the poroelasticity parameter to 0.8 . In the ensemble model, shown for comparison in Figure 6, a linear elastic response is assumed in the same pressure range.

Under high confinement, the cracks and joints are closed and effect of poroelasticity is reduced. Because the poroelasticity is caused by cracks and joints, an in situ rock mass which contains many joints should have an increased effect. Mesoscale modeling of a jointed rock (Vorobiev, 2008) showed that some features of the mechanical response of joints such as, strongly nonlinear stress-strain response in the stress range of 1-10 MPa and hysteresis in loading-unloading cyclic loading, are inherited by the rock mass. If joints are distributed nonuniformely, this may cause elastic anisotropy, because the poroelasticity associated wit the joints will work differently in various directions.

Thus, the joints present in the rock formation can cause both degradation of the effective strength as well as the reduction in the elastic wave speeds. The former is modeled using GSI strength scaling and the 
later is modeled by increasing the poroelasticity parameter, a. The poroelasticity will mostly effect the wave propagation in a far range where the normal stresses are not big enough to close the joints.

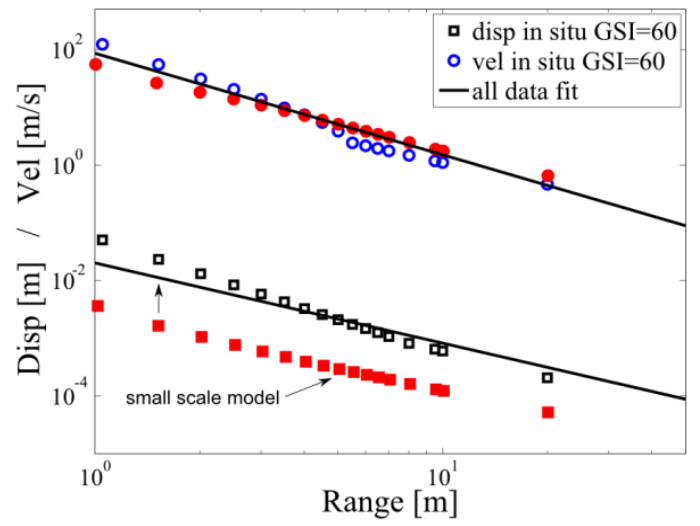

Figure 7. Peak displacement and velocity attenuations calculated in $1 D$ spherical explosion using the small scale model (solid squares and circles) versus the nuclear data fit. Open squares and circles show an in situ model calculations

The extended intact granite model described above presents an alternative to the ensemble model for in situ applications if appropriate scaling is used. Figure 8 shows comparison between the ensemble model (bold curves) and the current model (dashed lines- GSI scaled model, thin lines- the intact granite model) for a spherical explosion. The yield was equivalent to SPE1 explosion. It is seen from Figure 8, that GSI scaled model can produce waveforms much different from the intact granite model and similar to the ensemble model. 


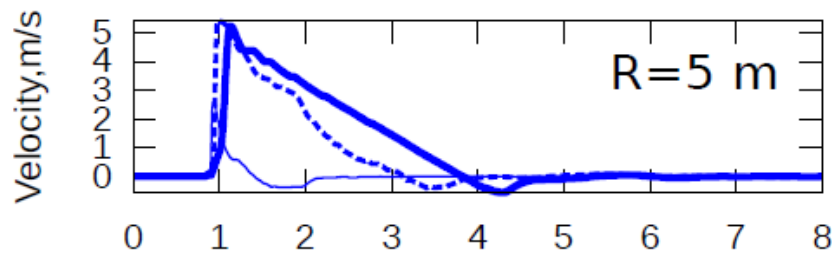

Time,ms

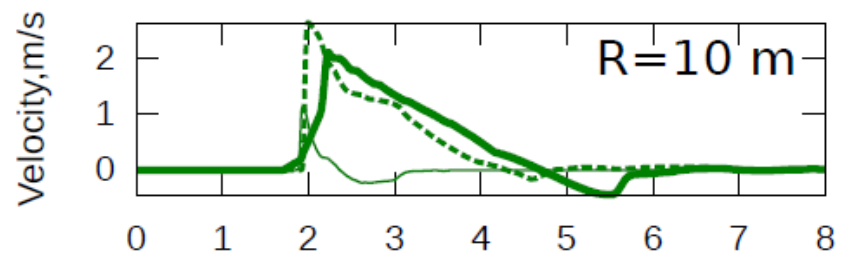

Time,ms

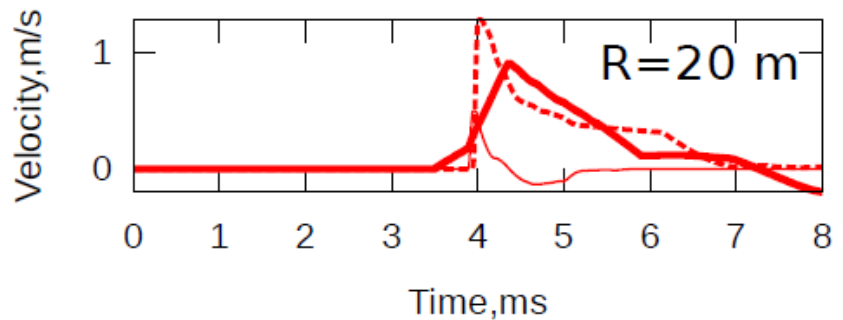

Figure 8. Radial velocities calculated for SPE1 test using various models: bold-ensemble model developed to fit nuclear data, thin lines-model built based on the intact samples collected from the site, dashed-continuum model built using guidance from the large scale simulations.

\section{DISCRETE MODELING AND JOINT EFFECTS}

GEODYN-L, a Lagrangian massively parallel hydrocode (Vorobiev, 2011) was used to study effects of joints by modeling them as contact interfaces. In the discrete simulations, the geologic medium is represented as an assembly of intact rock blocks separated by joints and fractures. As it was shown above, the intact rock was modeled using a constitutive model that accounts for the salient features of the response of geologic materials, and calibrated for granite using laboratory data, including data from core samples obtained from the SPE test bed. The joints were modeled as frictional interfaces with their own constitutive relations, which include governing equations for both frictional sliding along the joint surface as well as compressibility, or compliance, normal to the joint surface. The computational resources required in this case are formidable because of the large number of joints and the 3D character of the problem. Preliminary analysis done on smaller problems showed that the joint orientation and persistence play a big role in formation and attenuation of the waves. 
The jointed rock formation shown in Figure 9 was created by paving the space with parallelepiped blocks. The size of the region was $40 \times 40 \times 40 \mathrm{~m}$ centered around the source. Each block was subdiscretized by a number of elements with contacts set at the exterior faces. The properties of the contacts were varied randomly from sticky to weak contact (with low frictional coefficient, corresponding to the wet joint described above). This approach allowed us to describe a joint system with three main joint directions. These directions were identified from the experimental characterization of the joints at different locations.

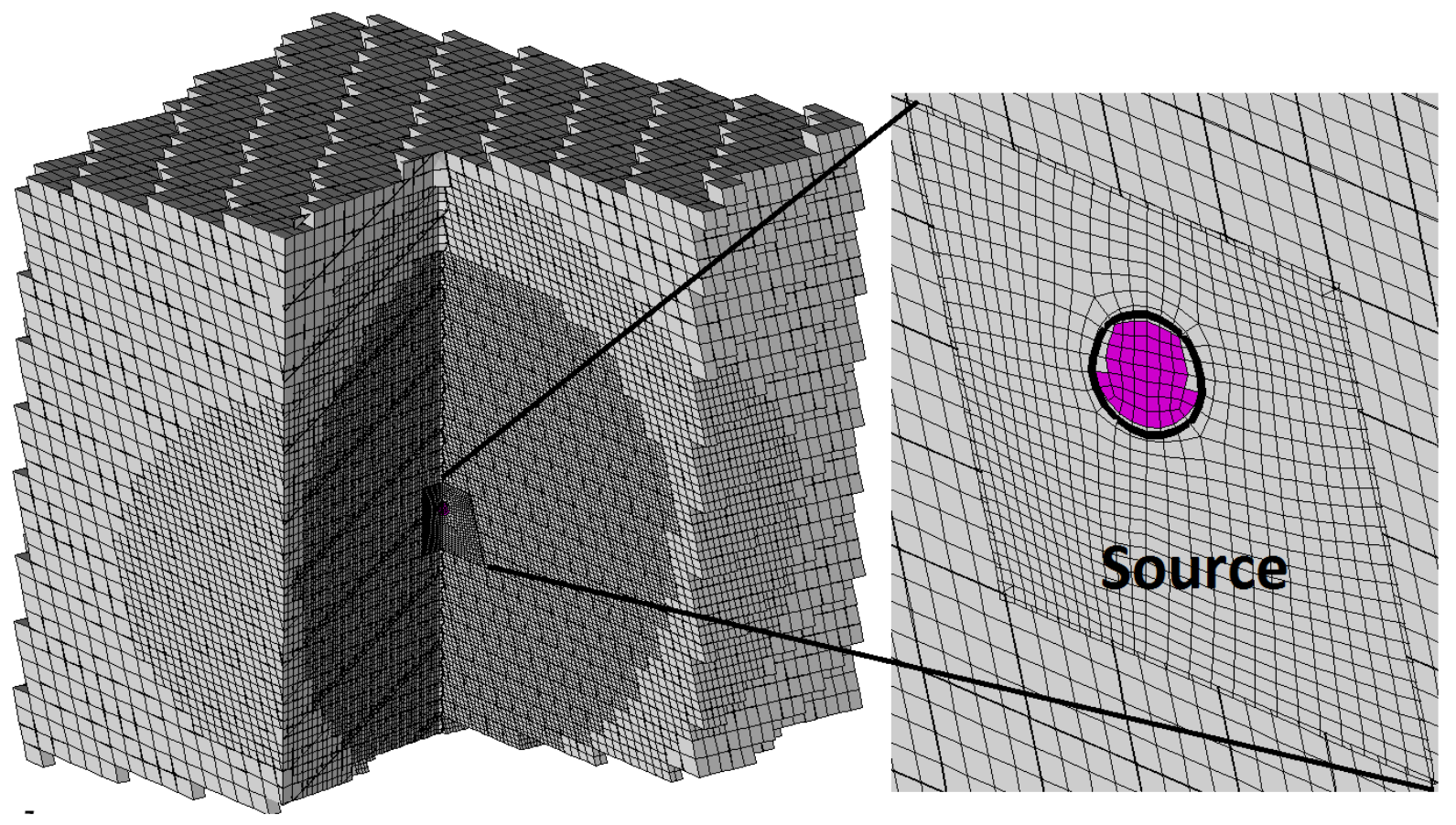

Figure 9. Computational mesh used for 3D study, the source block was meshed separately (shown on the left)

By decreasing the joint persistency an anisotropic wave turns into a symmetric wave which would be generated in an isotropic media. Figure 10 shows effect of joint persistency on the shape of the wave generated by the explosion.

Radial velocity at range $10 \mathrm{~m}$ in two different azimuthal directions are shown on plot $\mathrm{D}$, the plastic slip on the joints is shown on plot $\mathrm{C}$. 

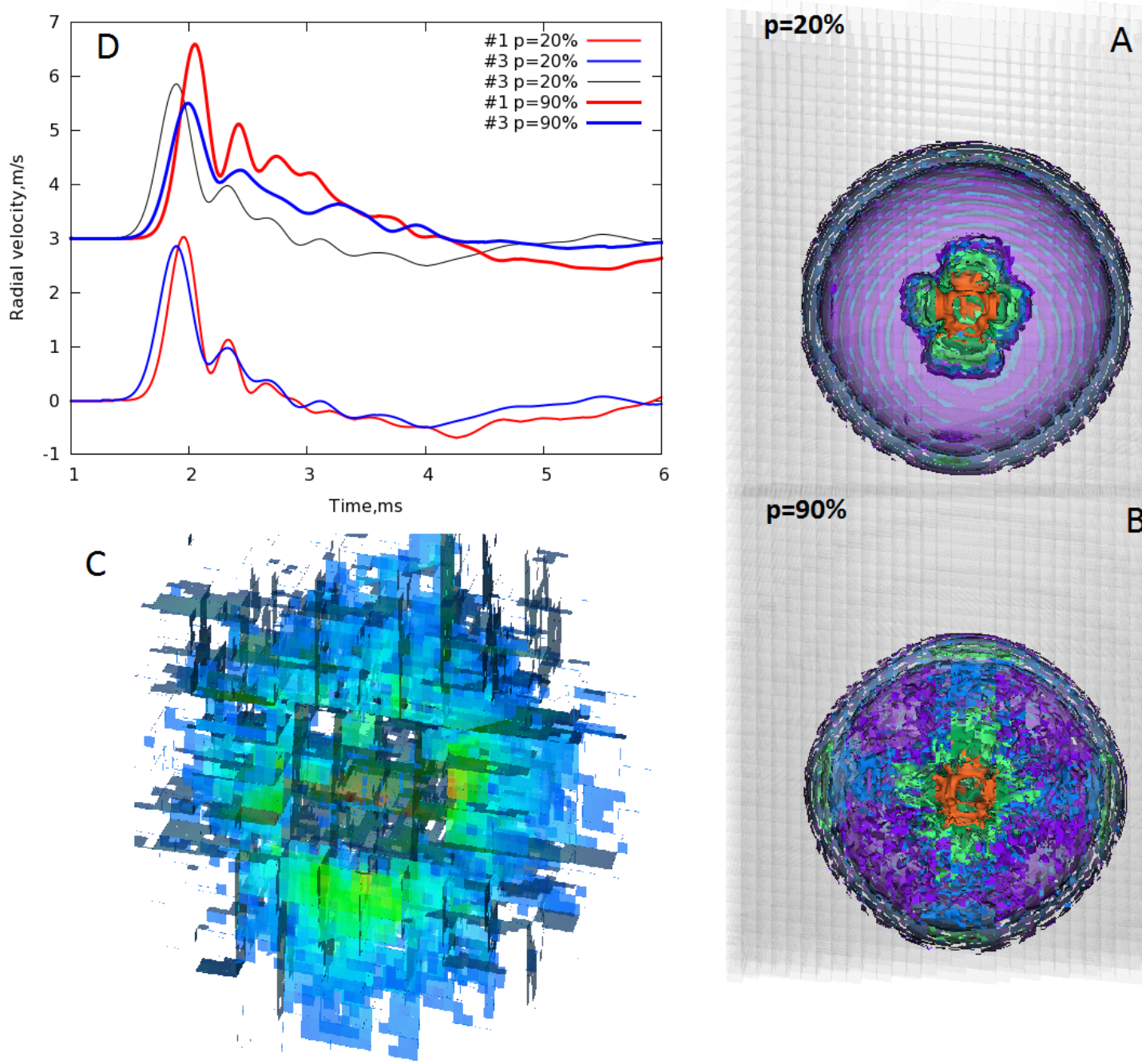

B

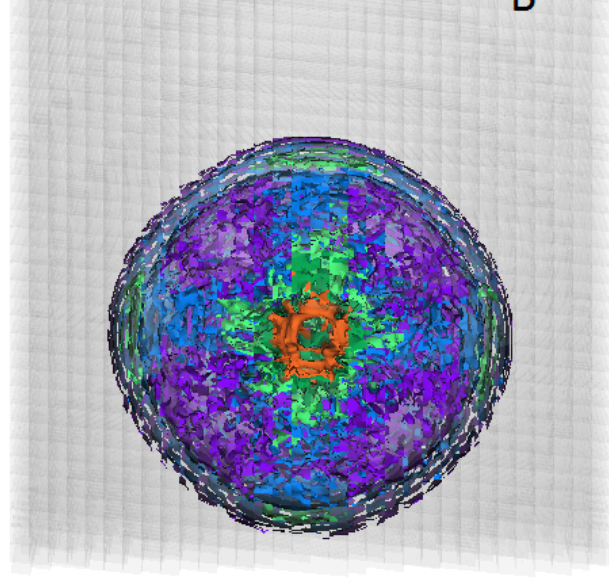

Figure 10. Effect of the joint persistency: Pressure contours (5-50 MPa) calculated for joints with different persistency $(A-20 \%, B-90 \%)$ at time $2.6 \mathrm{~ms}$, C-shear slip 0-10 mm calculated at the joint planes around the source, $D$ - Radial velocities calculated at gauge locations \#1 and \#3 at the rage $10 \mathrm{~m}$ in different azimuthal directions. 


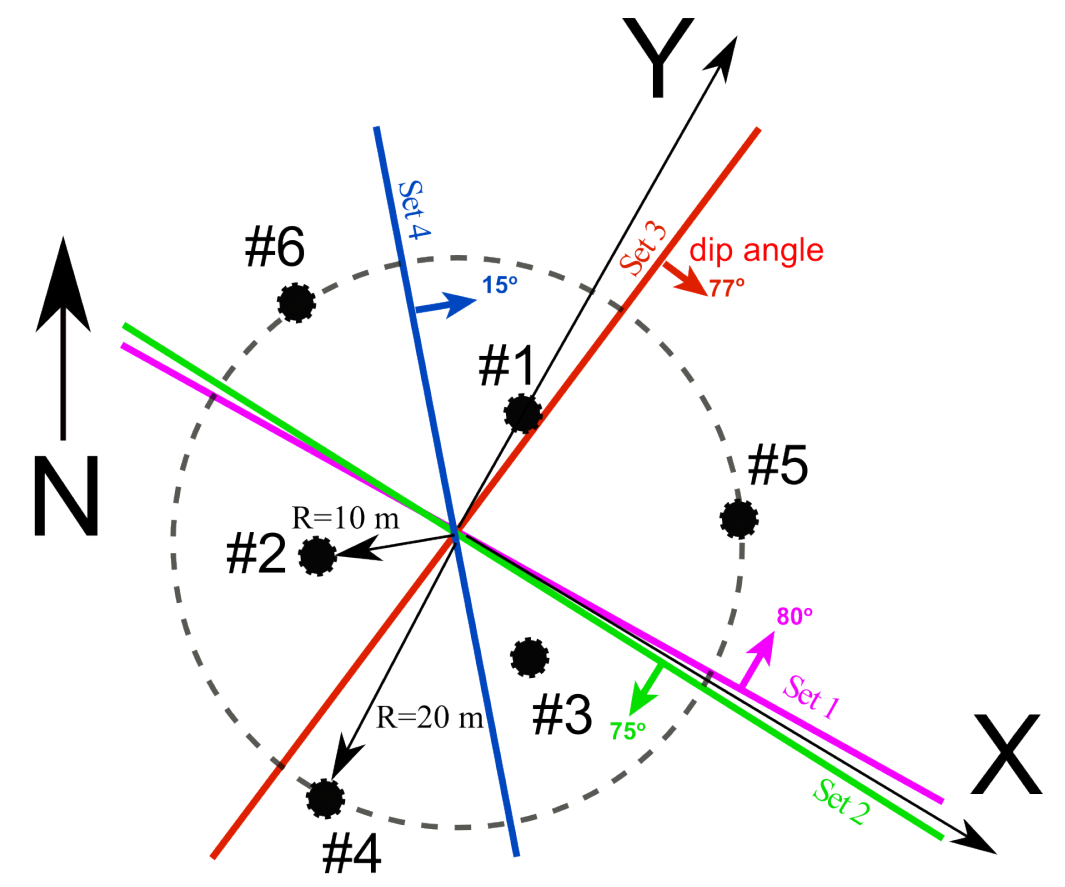

Figure 11. Joint dip directions, well locations and coordinate axis locations.

Figure 12 shows that in the case of the persistent joints more energy is flowing in the northern direction (to the left) rather than in the southern one due to wave interaction with joint Set 3 dipped at 77 degrees in south-east direction as it is shown in Figure 11. That may explain higher vertical velocity observed at the surface on the northern side. 

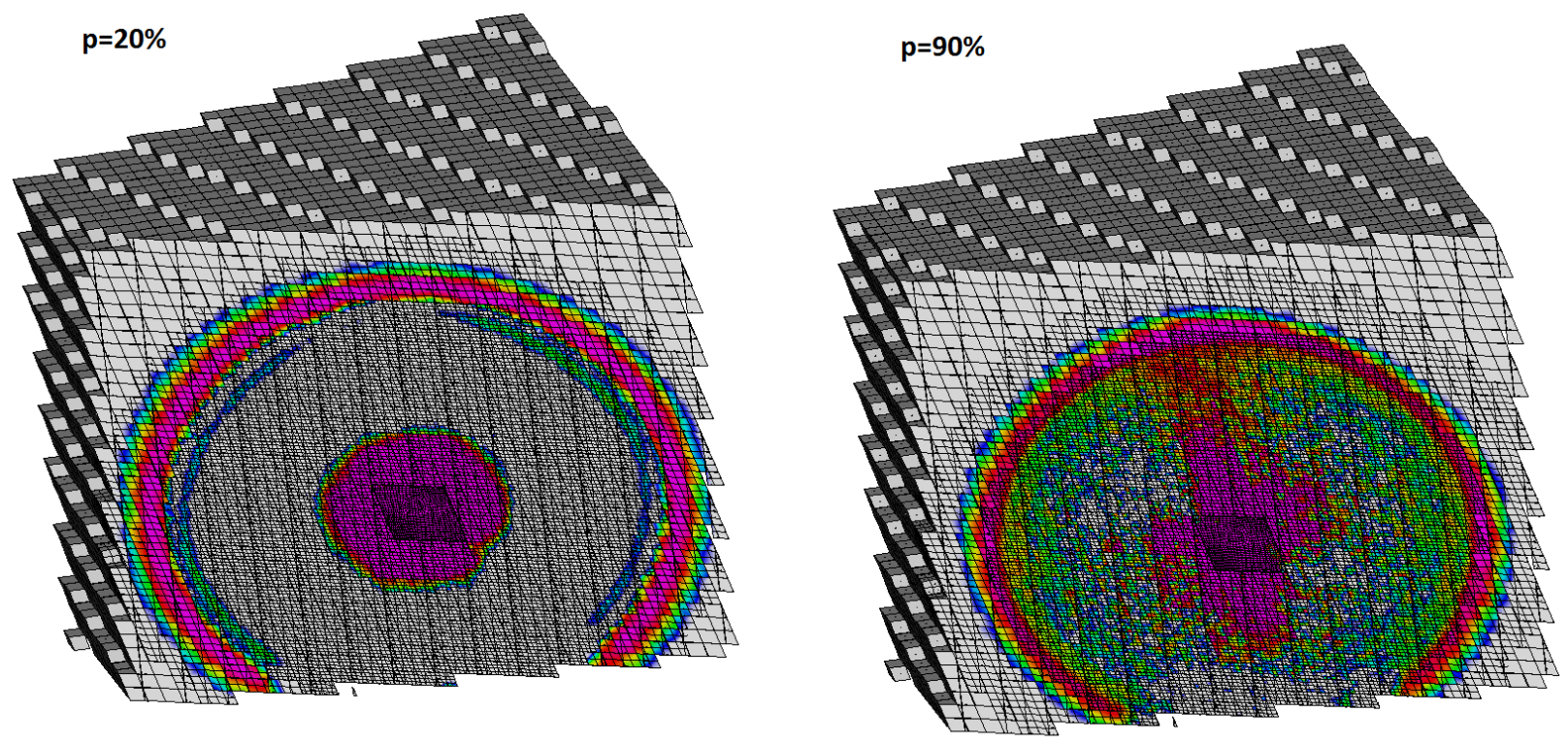

Figure 12. Cross sectional view in the eastern direction on the pressure contours (0-0.008 GPa) generated in jointed rock with different persistency (20\% on the left and $90 \%$ on the right)

Joint stiffness is important to overall impedance of the granite mass. Figure 13 shows that the calculated arrival times could be significantly delayed if the initial joint normal stiffness is reduced by an order of magnitude. Reducing the aperture at the same initial stiffness makes the joints more non-linear. It is interesting to note that peak velocities remain the same in this case even though the time of arrival is substantially reduced. Resolving a sub millimeter aperture could be problematic with mesh resolution $\sim 1$ $\mathrm{m}$ in some areas. We apply an advanced semi-implicit contact algorithm described in (Vorobiev, 2011) to reduce time step control by the joints. We see that the joints can make the waveform much wider but at the same time softening the joints effects the arrival time. 


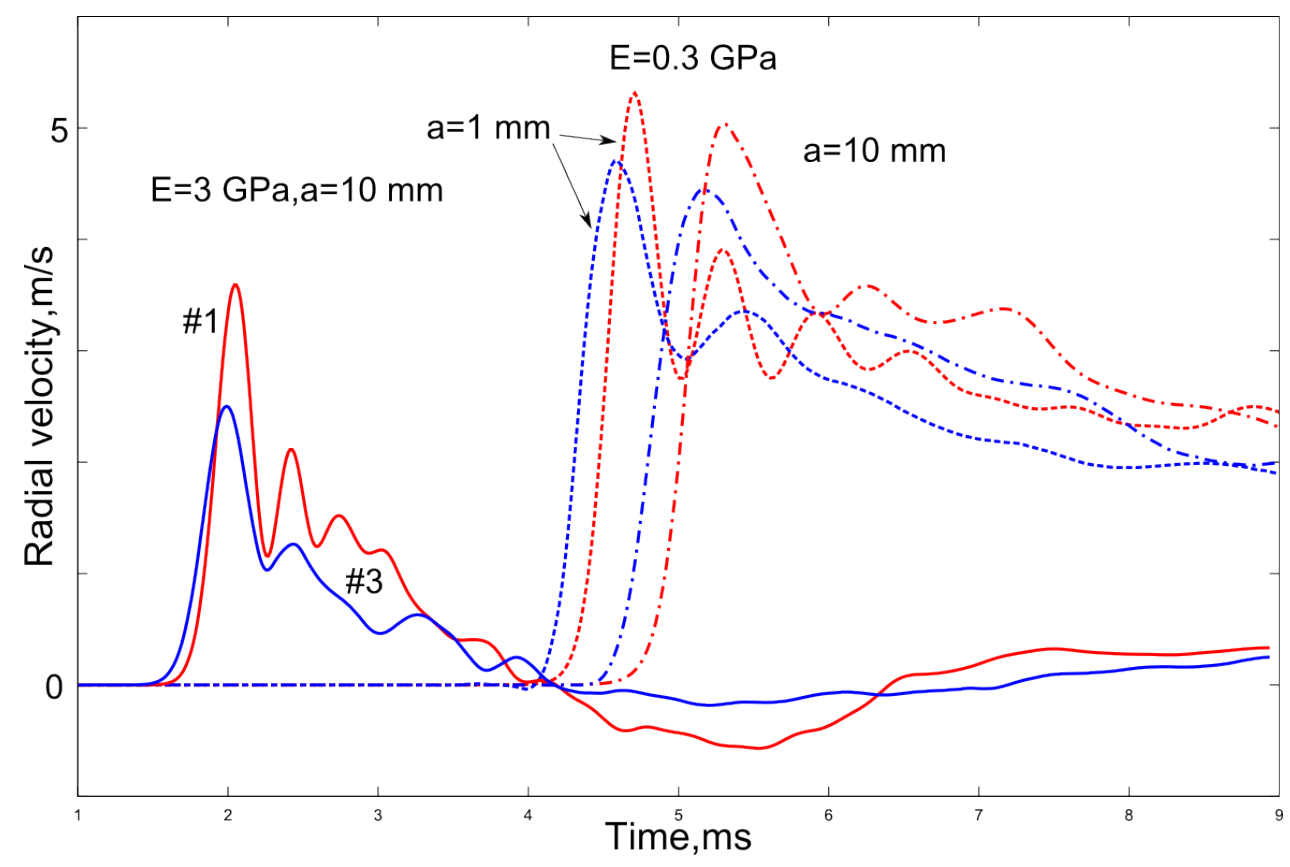

Figure 13. Effect of the joint initial stiffness and aperture on the waveforms and the arrival time at $10 \mathrm{~m}$ range: solid lines - stiffer contacts $10 \mathrm{GPa}$, dashed lines- softer contacts

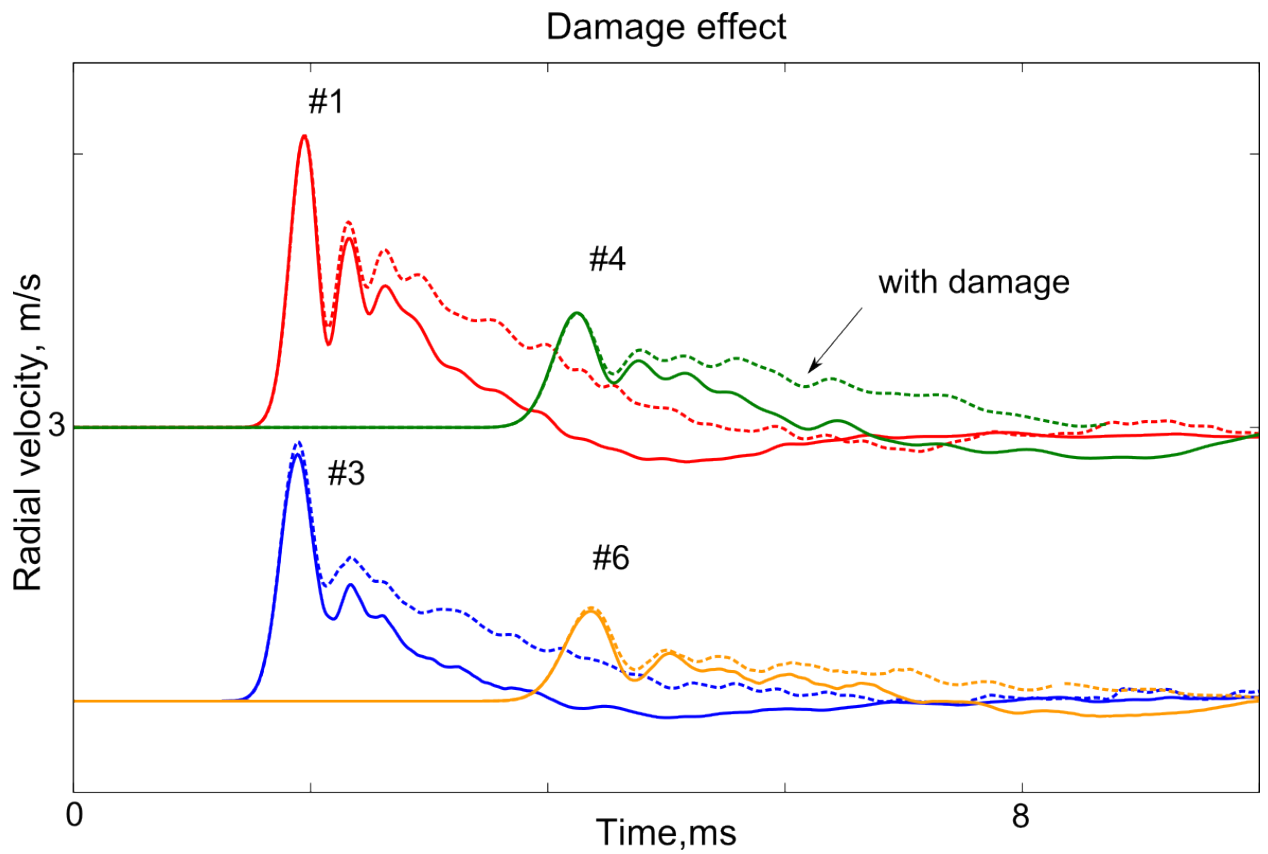

Figure 14. Effect of damage in granite blocks on the waveforms 
Discrete simulations done before assumed that the granite in the blocks remains intact. We have conducted an investigation to evaluate effect of damage inside the blocks on the waveforms. Figure 14 shows results of discrete simulations for radial velocities at some locations $(\# 1, \# 3, \# 4, \# 6)$ at the depth of the SPE3 explosion $(150 \mathrm{ft})$. When the granite was allowed to damage (dashed lines) wider velocity pulses at all locations were calculated.

\section{CONTINUUM Simulations OF SPE3 EVENT USING ENSEMBLE MOdEL}

\section{Simulation Method}

SPE 3 is designed to detonate in the same cavity excavated by SPE2 with the same yield (1000 $\mathrm{kg}$ TNT) to investigate the effect of the incurred damage on the wavefields. As for SPE2 we perform the predictions for SPE3 using the same 3D Eulerian code as for SPE2 with adaptive mesh refinement capabilities. The difference between the SPE2 and SPE3 prediction calculations lies in that the damage caused by SPE2 at the end is retained and serves as the initial condition for SPE3 to simulate the realistic scenario in the same source location. Also since SPE2 calculation predicted a cavity expansion to $\sim 1 \mathrm{~m}$ and radius of the SPE3 $25761 \mathrm{~b}$ explosive is about $0.6 \mathrm{~m}$ as in SPE2, the gap between the SPE2 cavity wall and the SPE3 explosive is filled with grout. An example of the damage sustained by SPE2 is shown in Figure 15 which is used the initial condition for SPE3. 


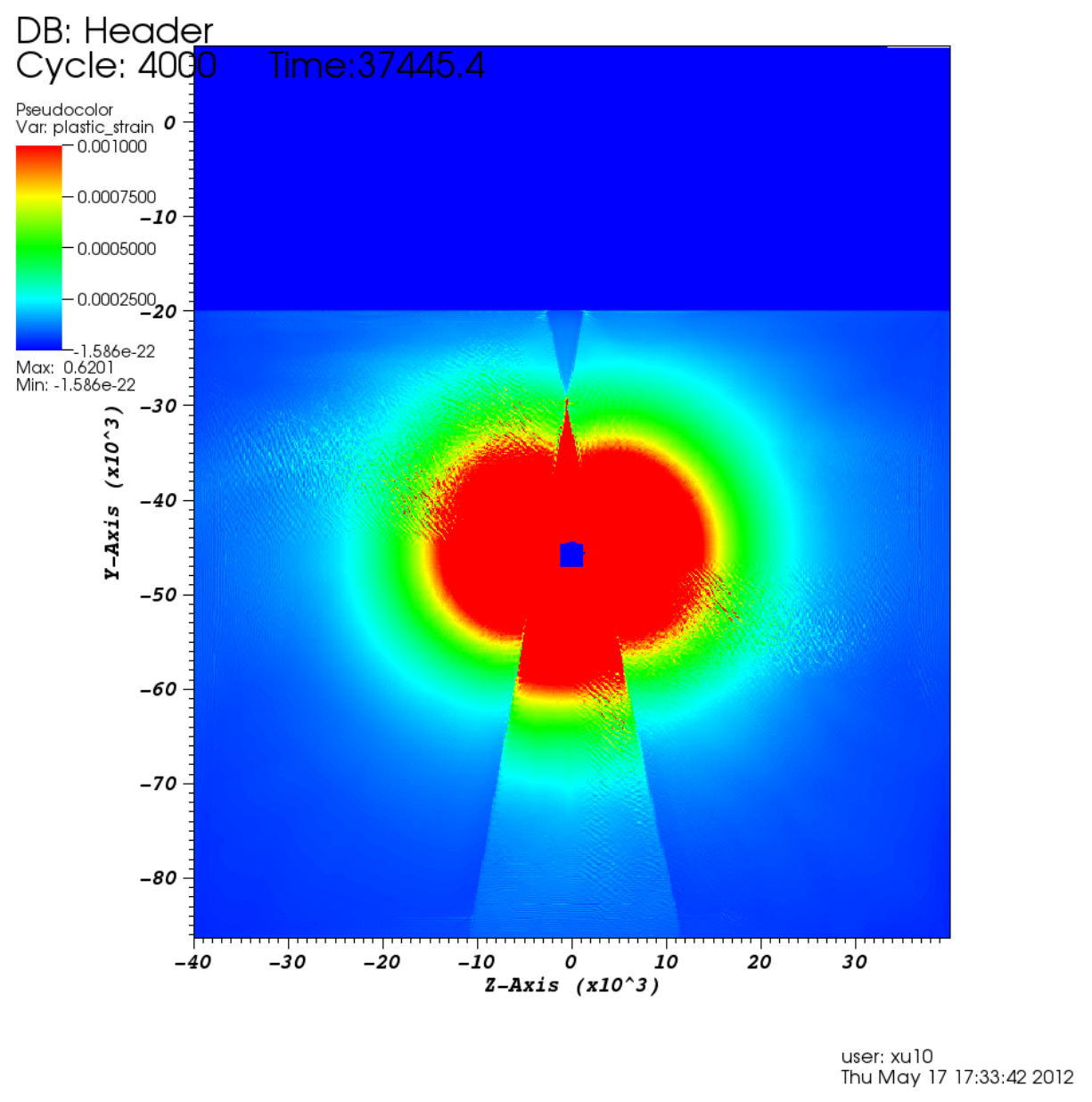

Figure 15. Damage sustained by SPE2 and serves as the initial condition for SPE3. The central blue box is the SPE3 charge and grout region. The top $20 \mathrm{~m}$ (from depth 0 to -20m) layer is the weak granite model and the damage zone is not plotted. The two fault locations are seen.

\section{Three-dimensional simulations of SPE3}

Similar to SPE2, we performed the predictions for the cases with and without the faults. We performed 2D and 3D calculations for validation without faults. To simulate the effect of the two faults we used the same strategy as in the SPE2 predictions by using a weak porous material representing the faults and by using the level set method to represent the fault surfaces. For the level set method two friction angles are used, 0 or 20 degrees as used in SPE2.

We calculate 5 solutions for SPE3, that are solutions without faults in $3 \mathrm{D}$, with faults using porous material model, with faults using the level set method representing the faults (with friction coefficients of 0 and 0.36 ) and one 2D solution without faults. Note at the distant hole \#11 only 2D solutions are 
provided at the three depths since the 3D grid setup for SPE2 did not cover that region. The results in 2D at \#11 are more complicated especially at the depth of 50ft due to the surface damage complexities and the large incidence angle. Again generally as seen in the SPE2 predictions, we see the radial motions are the dominating components at all the gage locations and the transverse components are relatively small. It is seen that the 5 solutions are quite consistent with each, indicating that the effects from the two faults are not significant on the wave motions in the near field.

We present the differences between the SPE2 and SPE3 at borehole \#4 in Figure 16 as an example to show the effect of the sustained damage on the outward motions in our predictions with our granite model. The level set method with a zero friction is used in this comparison. The red lines represent the SPE3 velocity waveforms and the green lines the SPE2 waveforms at $3 \mathrm{depths}$ (50ft, 150ft and 180ft). It is seen that the wavefields are again dominated by the radial motions in the SPE3 scenario just as in SPE2 and transverse components are still relatively small. The amplitudes for SPE2 and 3 are quite similar, implying the damage zone around the cavity impose little extra attention on the outgoing wavefields. However we notice that 1) the pulse widths seem to be reduced by the damage zone, indicating the total energy emitted to the far field is tempered by the damage sustained by SPE2; 2) the shock waves arrive slightly earlier from SPE3 than from SPE2, likely due to the fact that the material has been compacted by SPE2 in the near field and the apparent material strength seems stronger from our model. Implemented for predictions of SPE1 and SPE2, the model might need to include somewhat strength reduction due to damage after the SPE3 data are available. 

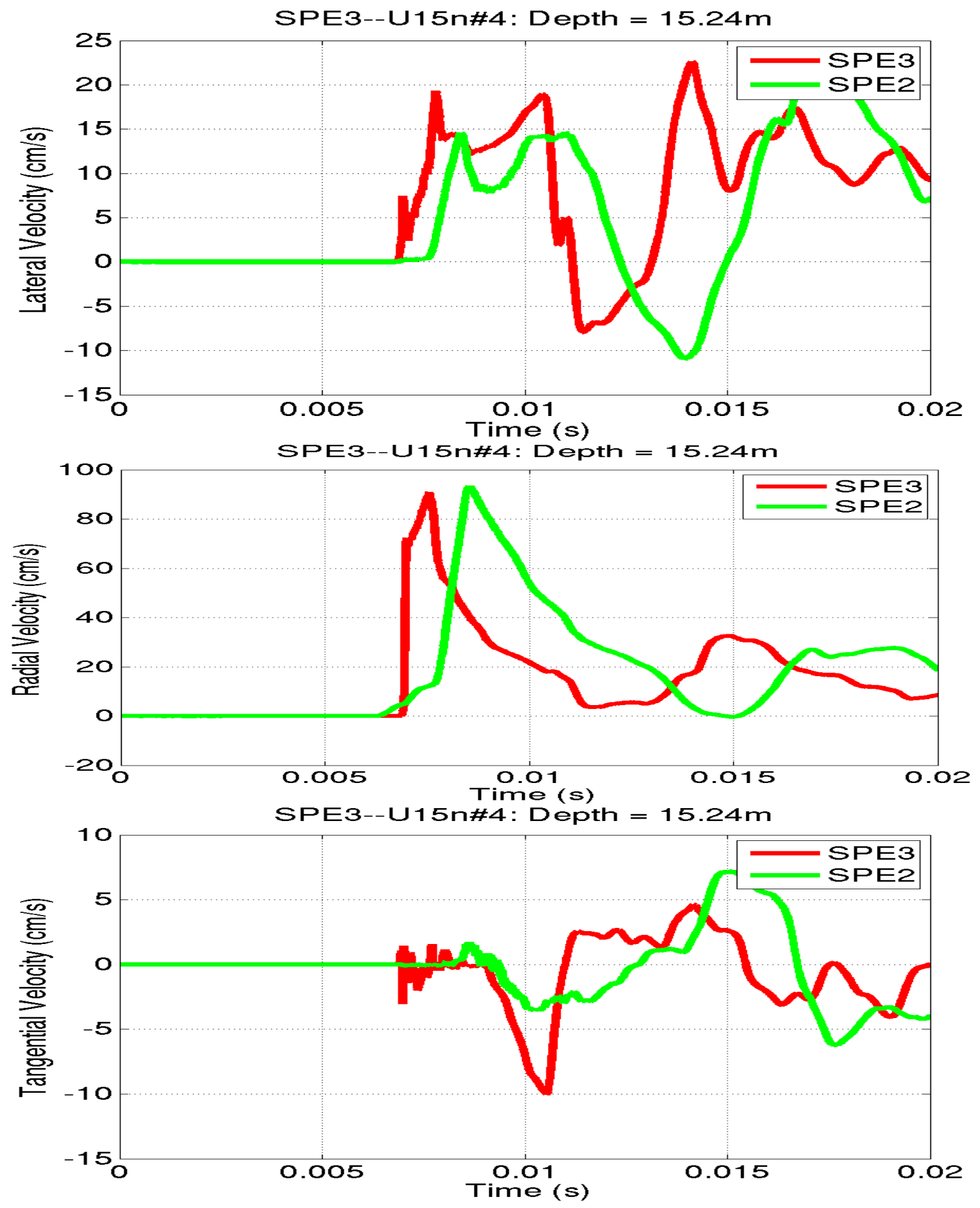


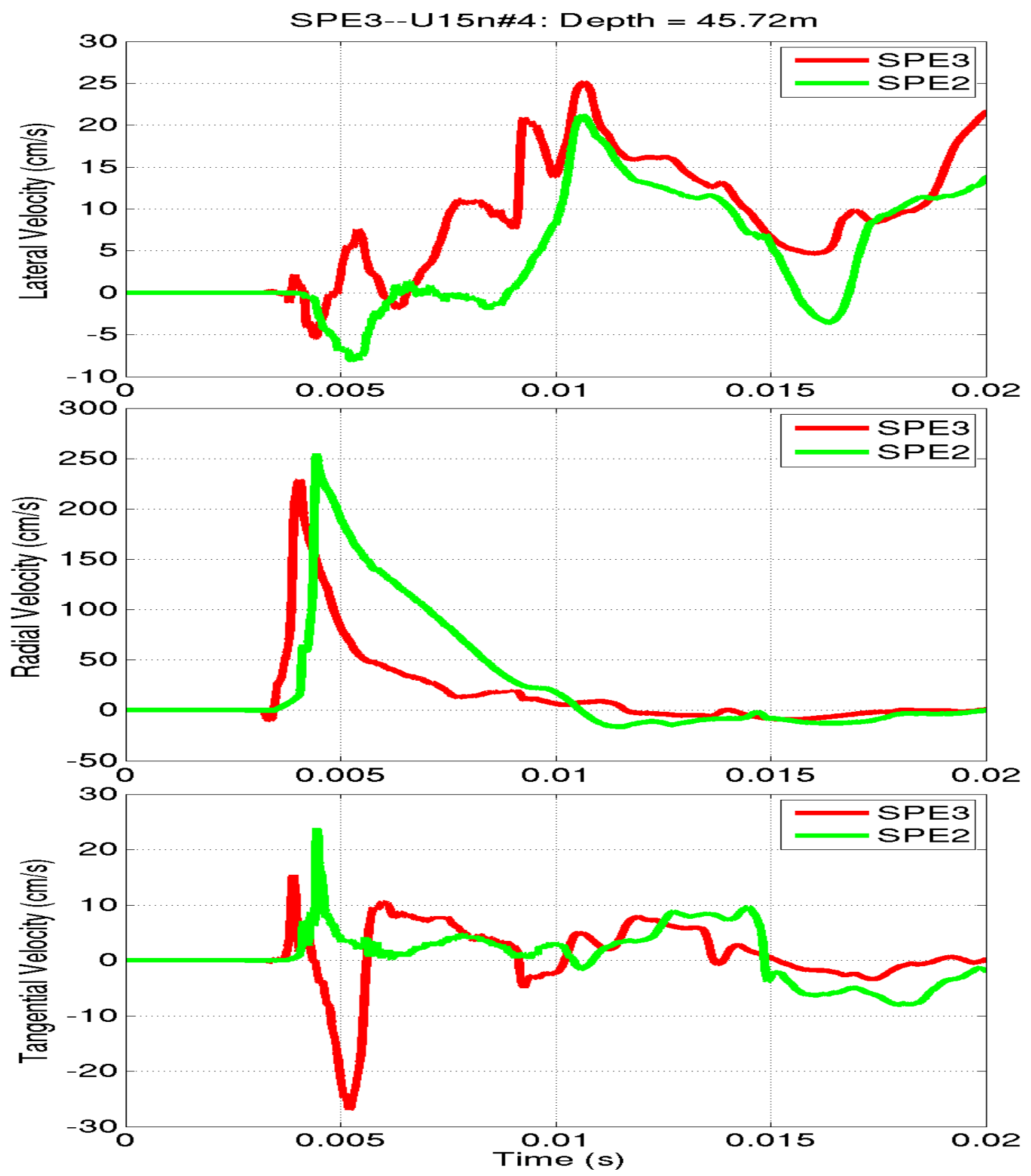



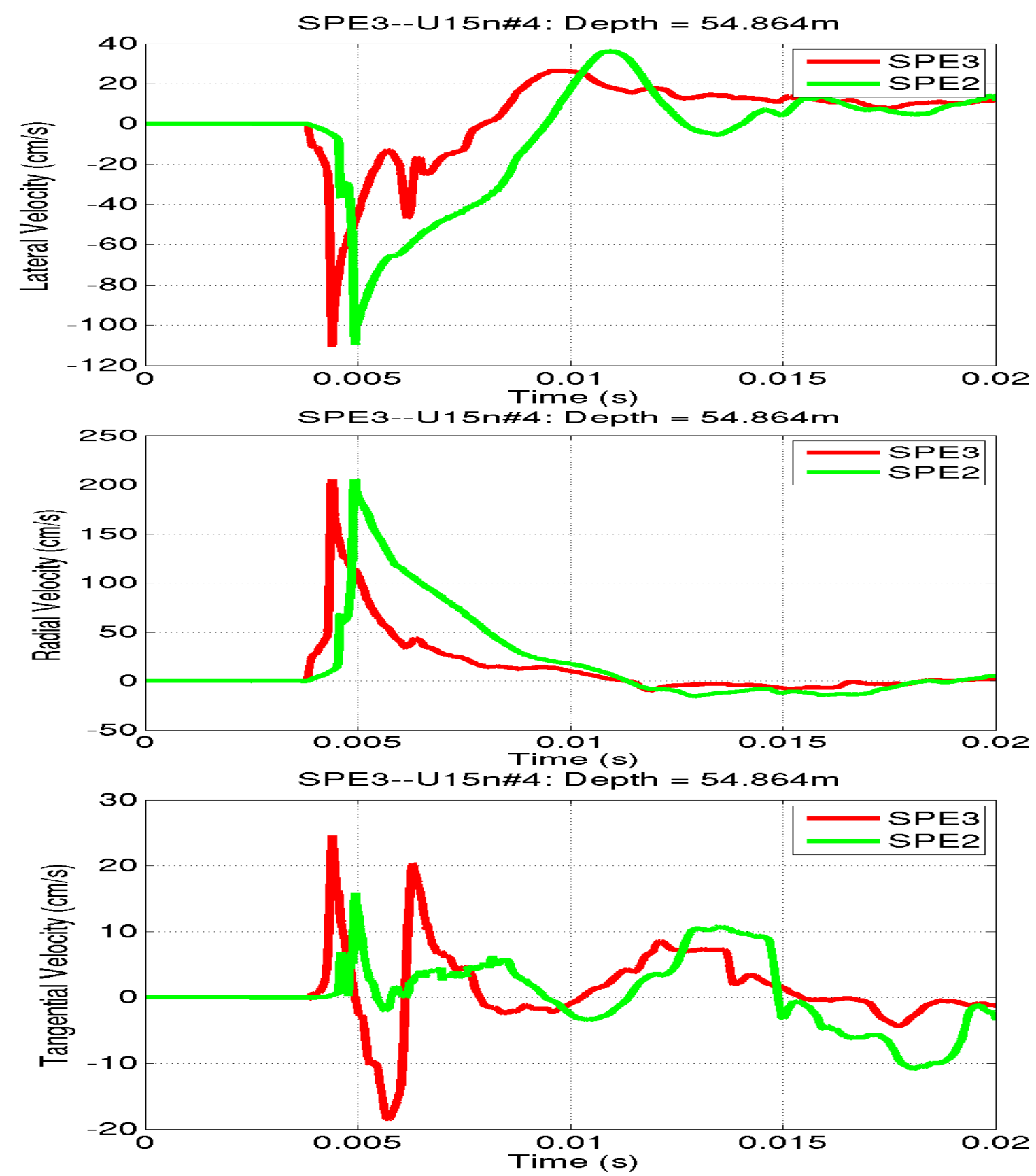

Figure 16. Comparison between SPE2 (green) and SPE3 (red) predictions at 3 depths (50ft, 150ft and 180ft) in borehole \#4. Three velocity components are plotted. The dominant wave motions are radial from the explosion for both explosions. 
Figure 17 shows the resulting damage slice from SPE3 after SPE2. The level set method with a zero friction was used. Compared with Figure 15 of the resultant SPE2 explosion-induced damage, the damage zone by SPE3 is seen enlarged slightly, consistent with the waveform conformality seen in Figure 16. The damage in the top weak layer is not plotted. Again as seen in the SPE2 simulations the damage zones are seen not symmetric either in SPE3 but the similarity on the waveforms at different azimuths indicates the effect of the faults is likely to be minimal.

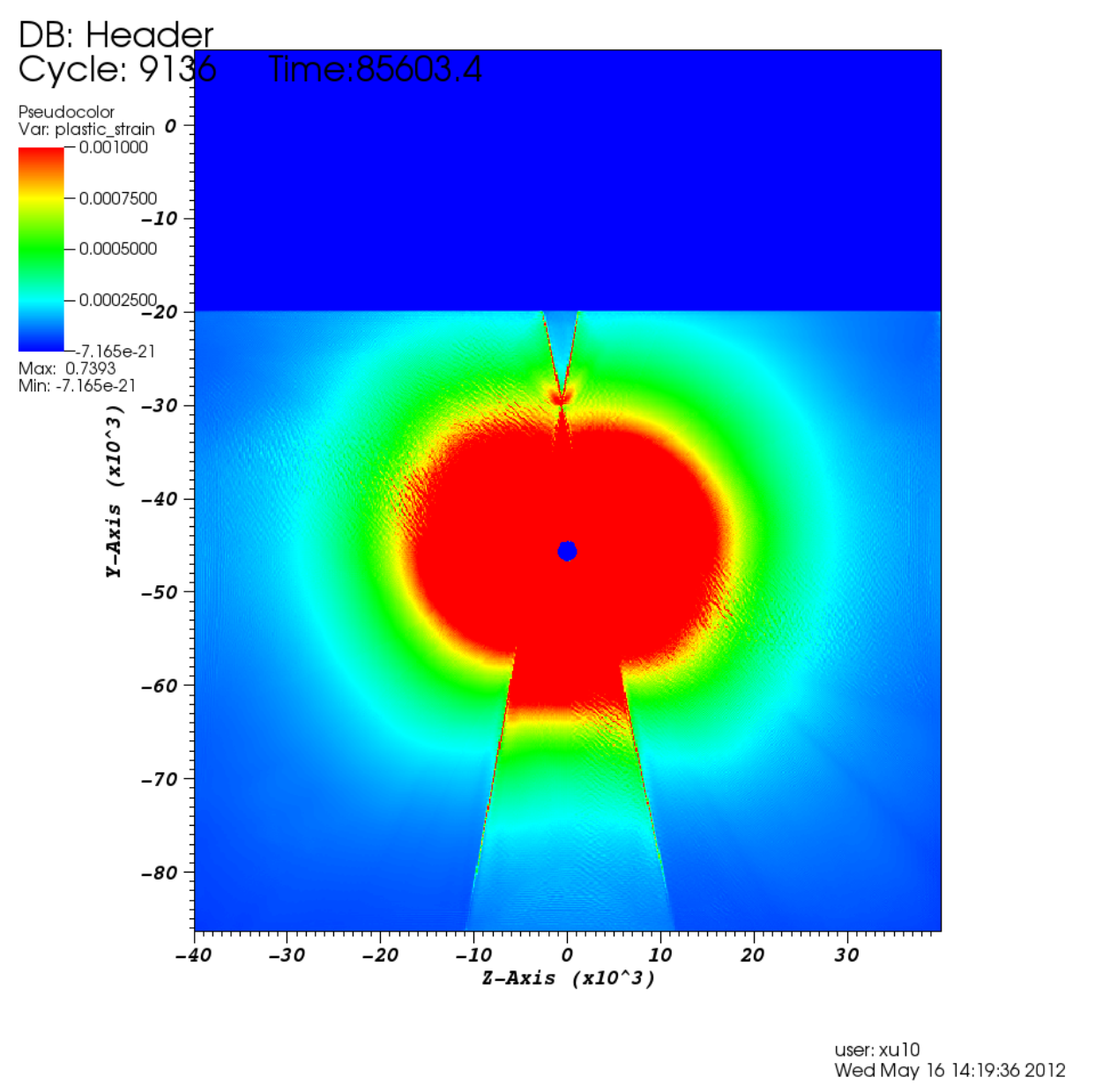

Figure 17. Induced damage by SPE3 after SPE 2. 
Similar as for SPE2, we compare the wavefields across the two fault planes at the four pairs (A-D) of locations at the shot level (150ft) shown in Figure 18. The results show in Figure 19 - Figure 22 in which the radial, tangential, lateral and total components are plotted for comparison and in Figure 23 - Figure 26 in which the components relative to the fault surface and the total component are plotted for comparison. The simulation results are calculated using the level set method with a zero friction coefficient on the fault plane. The radial, tangential and lateral components (Figure 19 - Figure 22) show slight variations across the faults even in this extreme case with perfect sliding condition on the fault surface and further confirm that the faults exert little influence on the outgoing waves. The 3 components in Figure 24 - Figure 26 show similar results across the faults. Such predictions are consistent with those for SPE2.

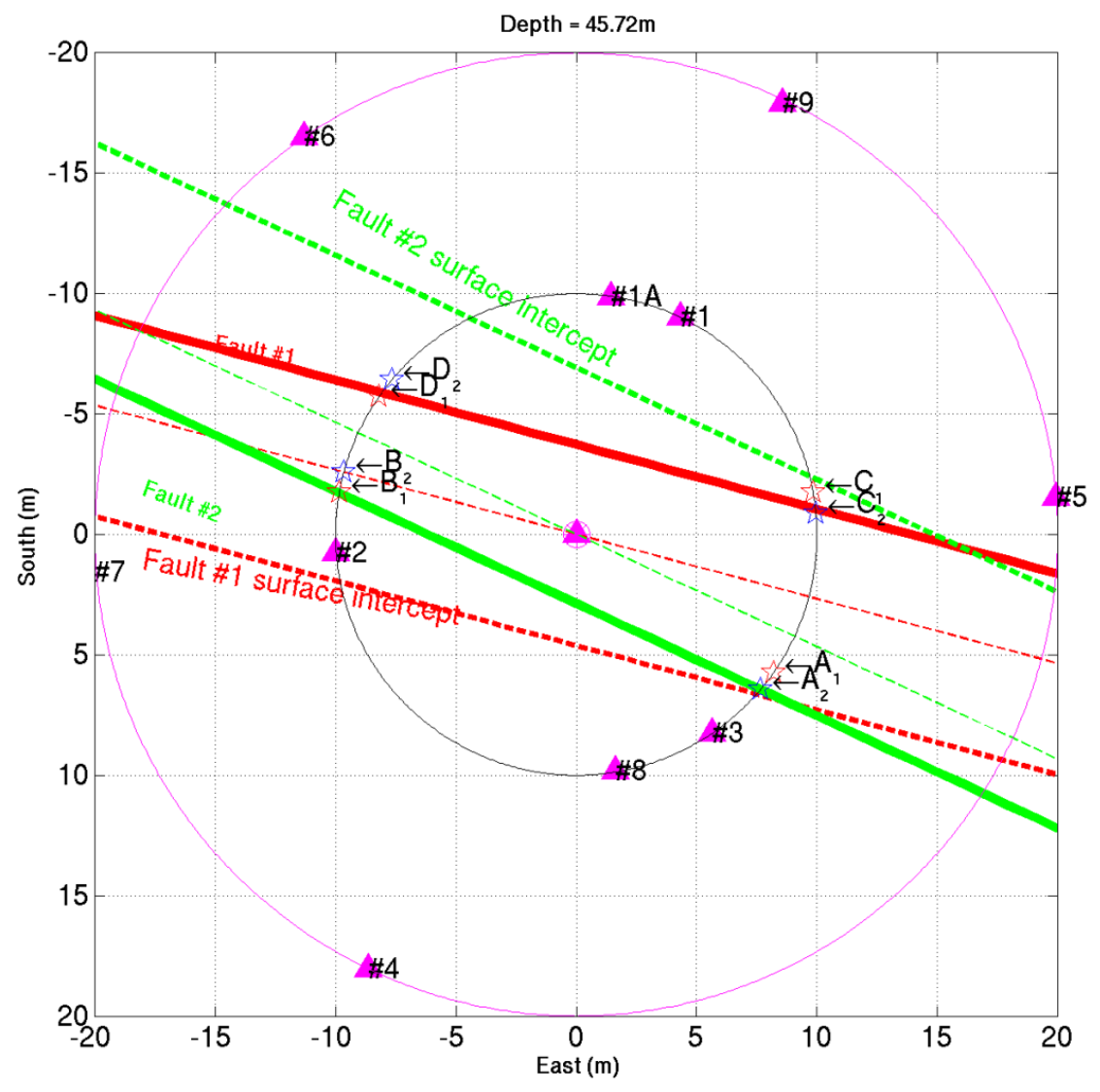

Figure 18. Four pairs of station location across the two faults at the SPE3 shot depth 

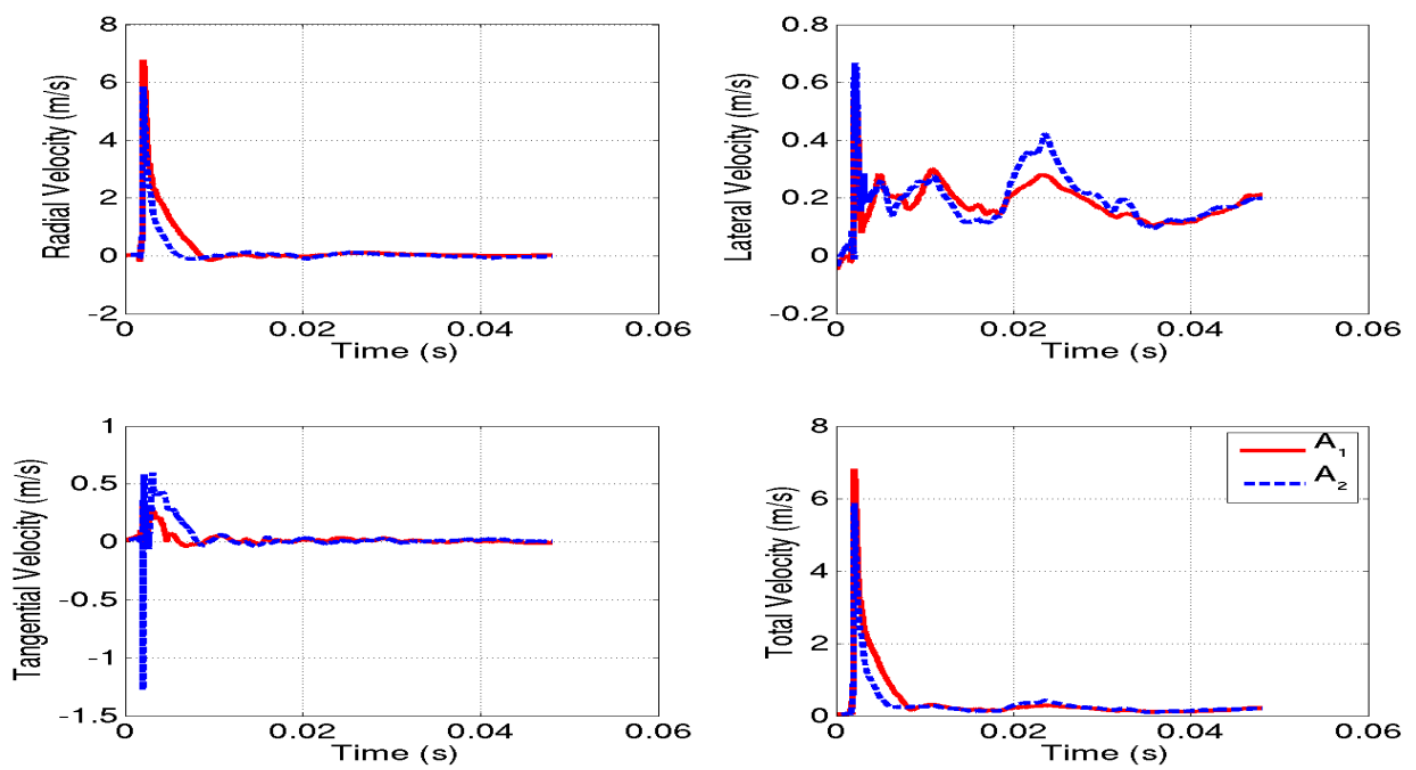

Figure 19. Comparison of wavefields across the faults planes at locations A1 and A2. The results obtained shown are obtained from simulations where the level set method is used to model the faults surface.
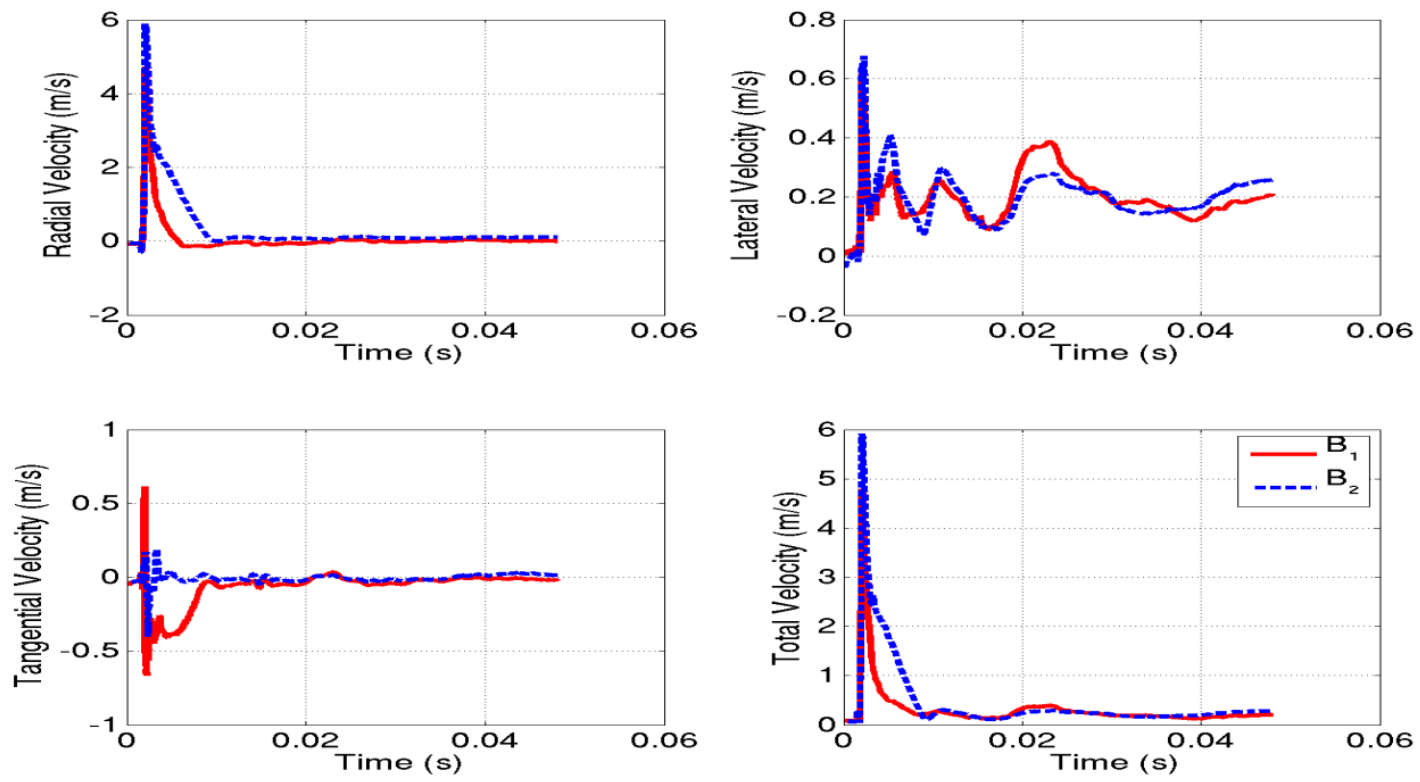

Figure 20 Comparison of wavefields across the faults planes at locations B1 and B2. The results obtained shown are obtained from simulations where the level set method is used to model the faults surface. 

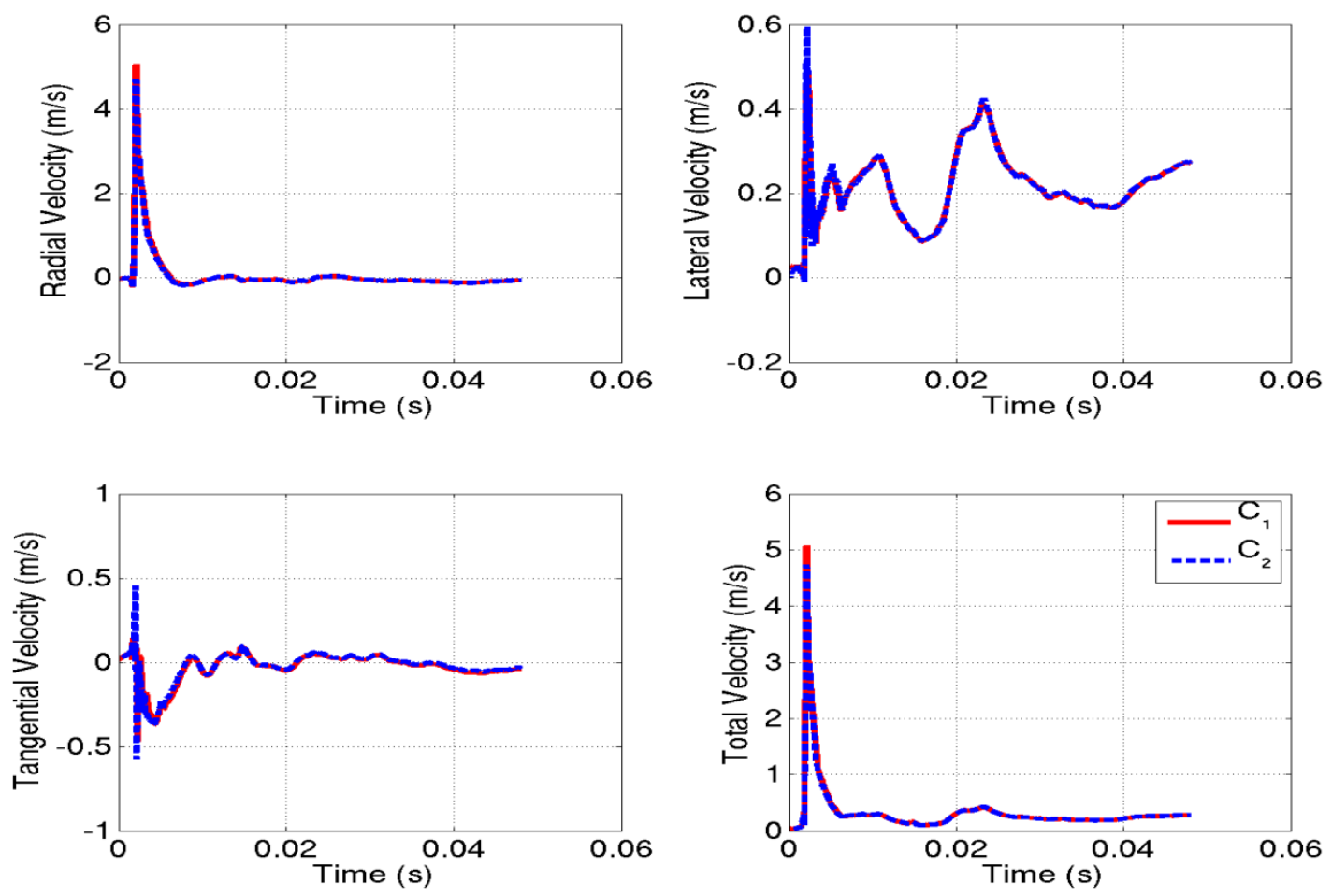

Figure 21. Comparison of wavefields across the faults planes at locations $C 1$ and C2. The results obtained shown are obtained from simulations where the level set method is used to model the faults surface. 

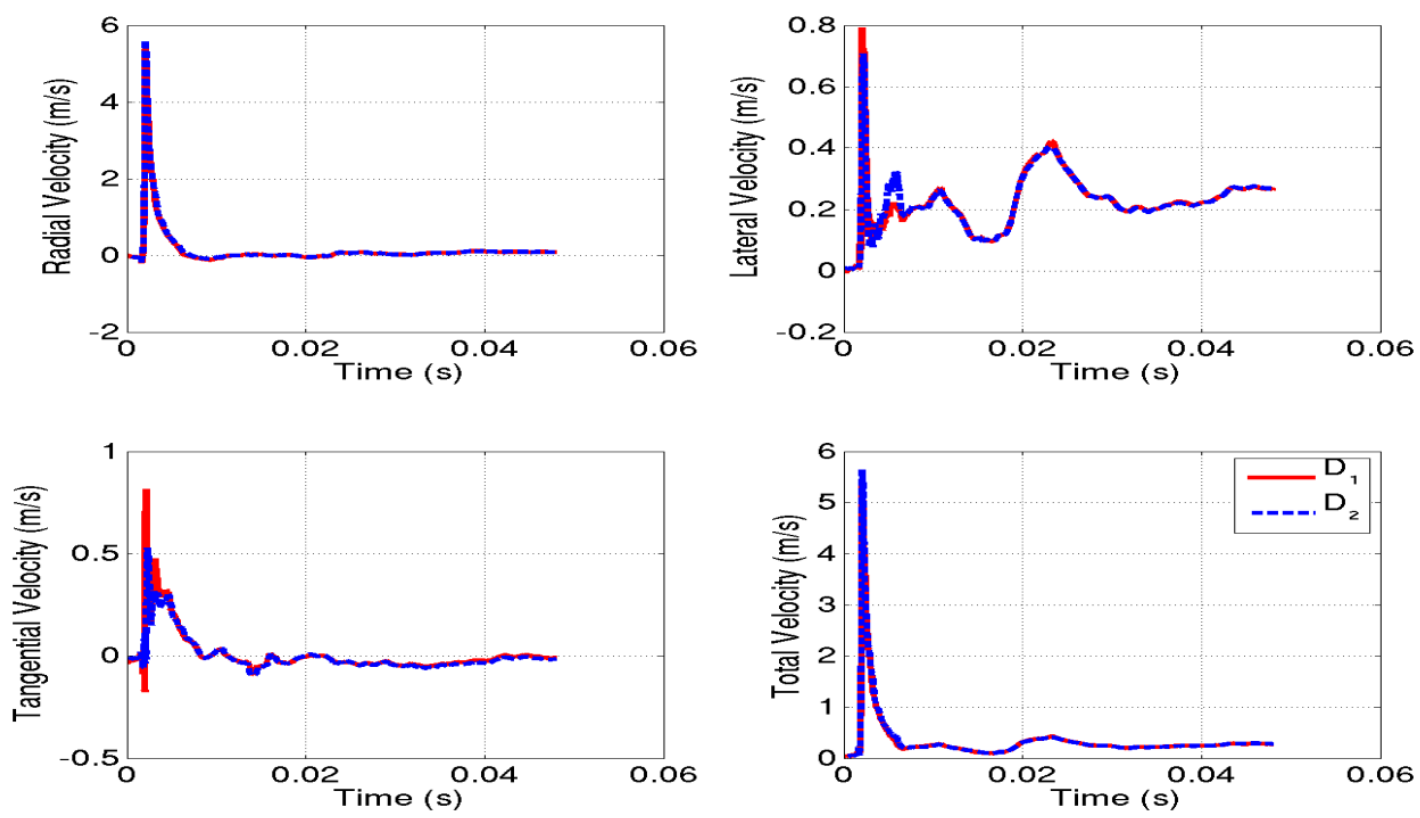

Figure 22. Comparison of wavefields across the faults planes at locations D1 and D2. The results obtained shown are obtained from simulations where the level set method is used to model the faults surface. 

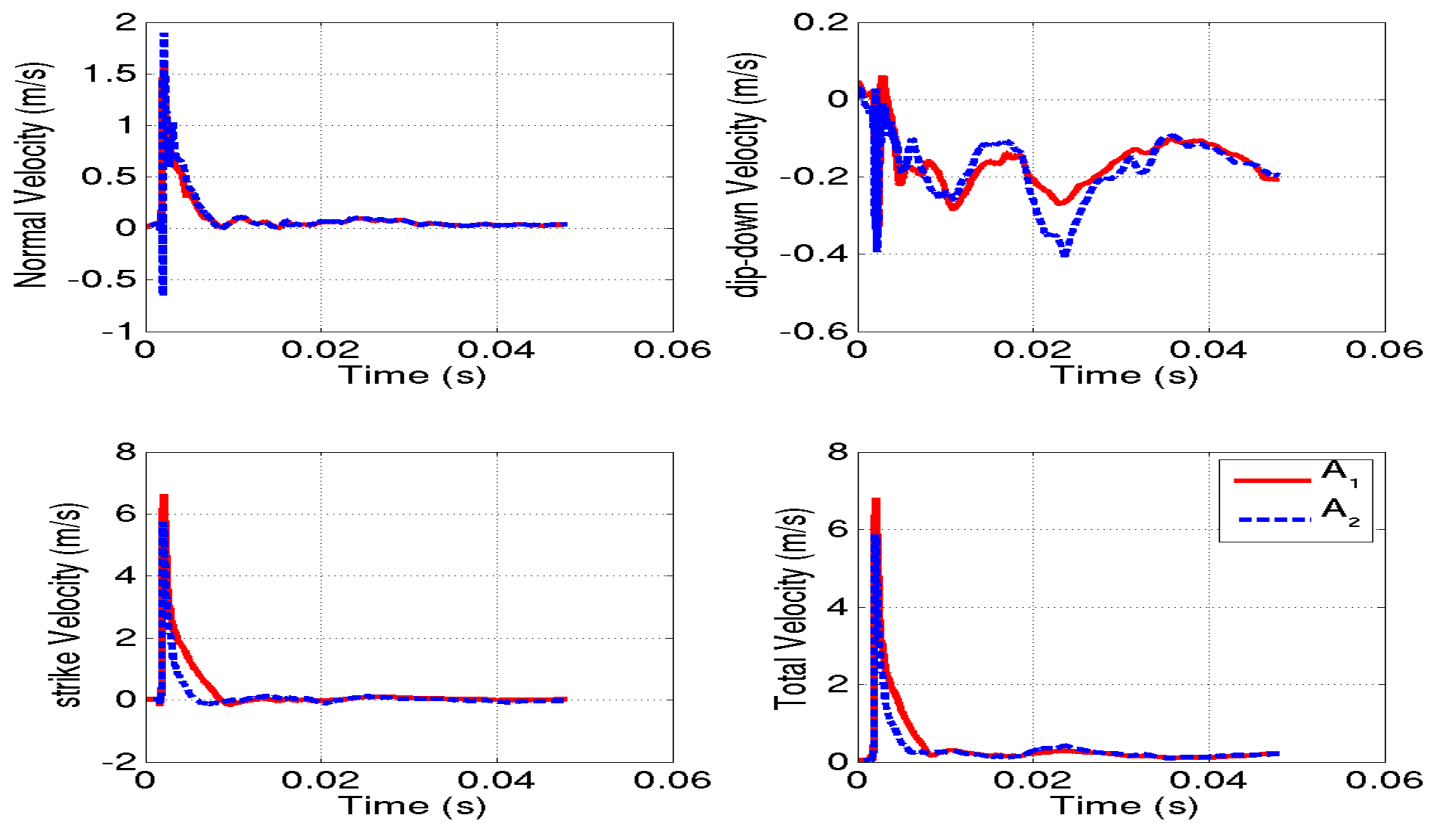

Figure 23. Comparison of wavefields across the faults planes at locations A1 and A2. Velocity components normal to the fault, along the strike and dip directions are shown. The results obtained shown are obtained from simulations where the level set method is used to model the faults surface. 

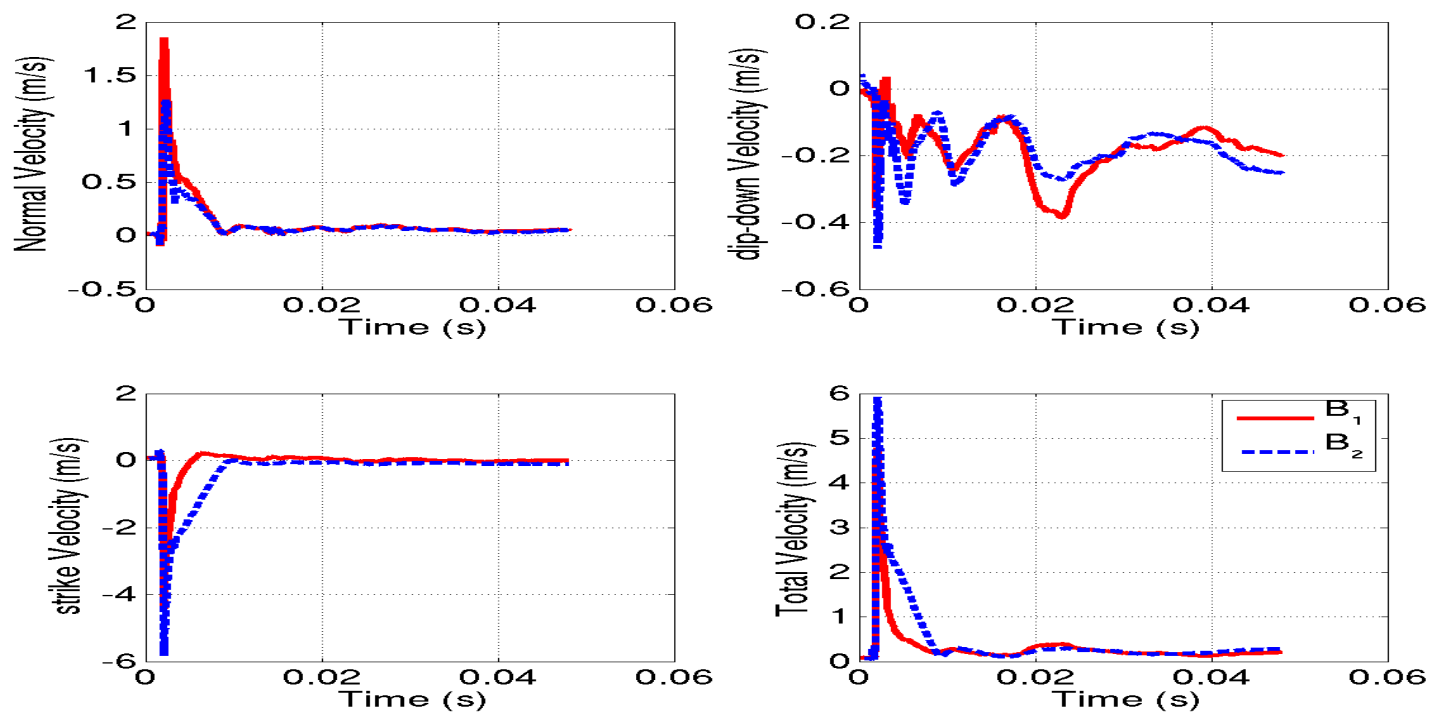

Figure 24. Comparison of wavefields across the faults planes at locations B1 and B2. Velocity components normal to the fault, along the strike and dip directions are shown. The results obtained shown are obtained from simulations where the level set method is used to model the faults surface.
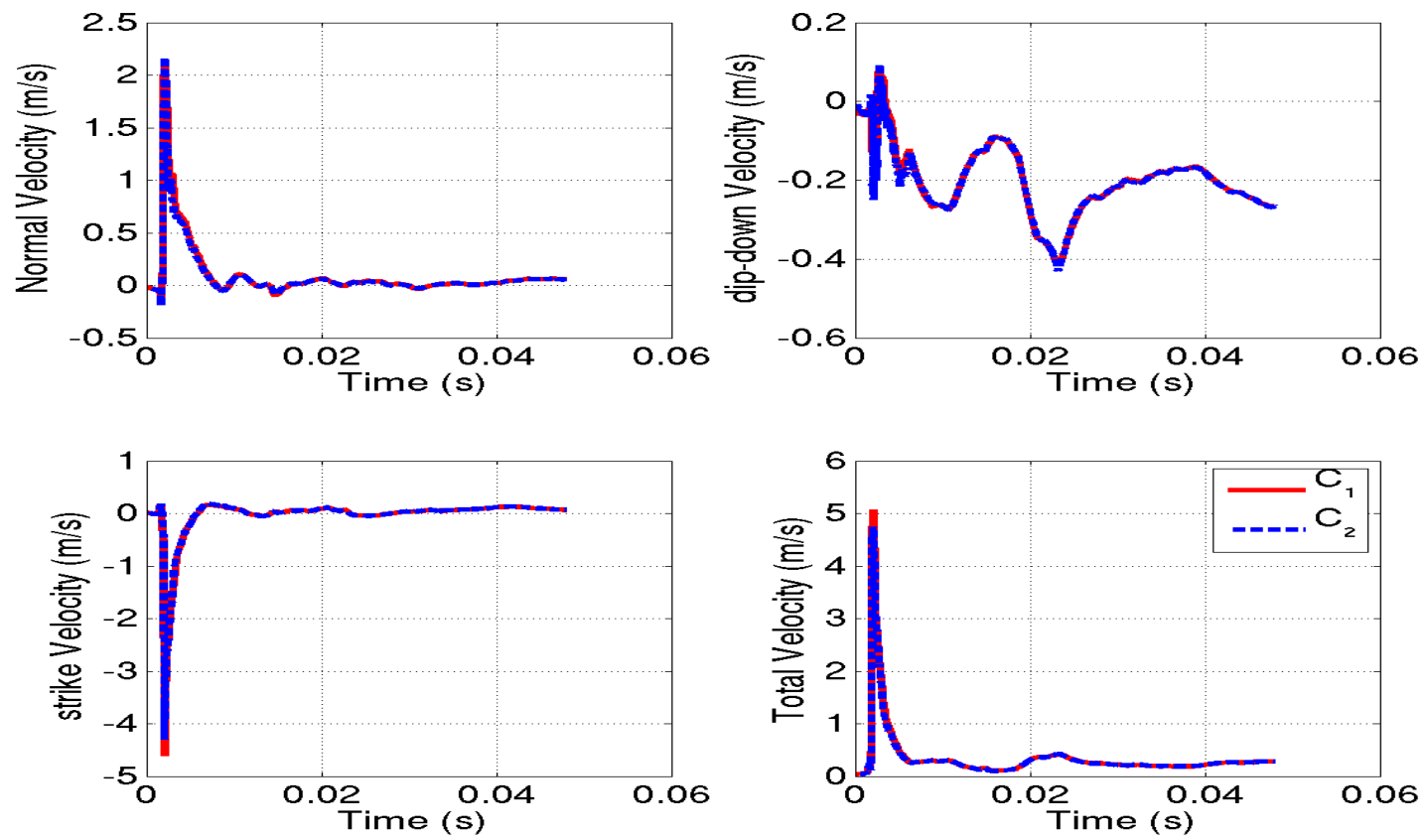

Figure 25. Comparison of wavefields across the faults planes at locations $C 1$ and C2. Velocity components normal to the fault, along the strike and dip directions are shown. The results obtained shown are obtained from simulations where the level set method is used to model the faults surface. 

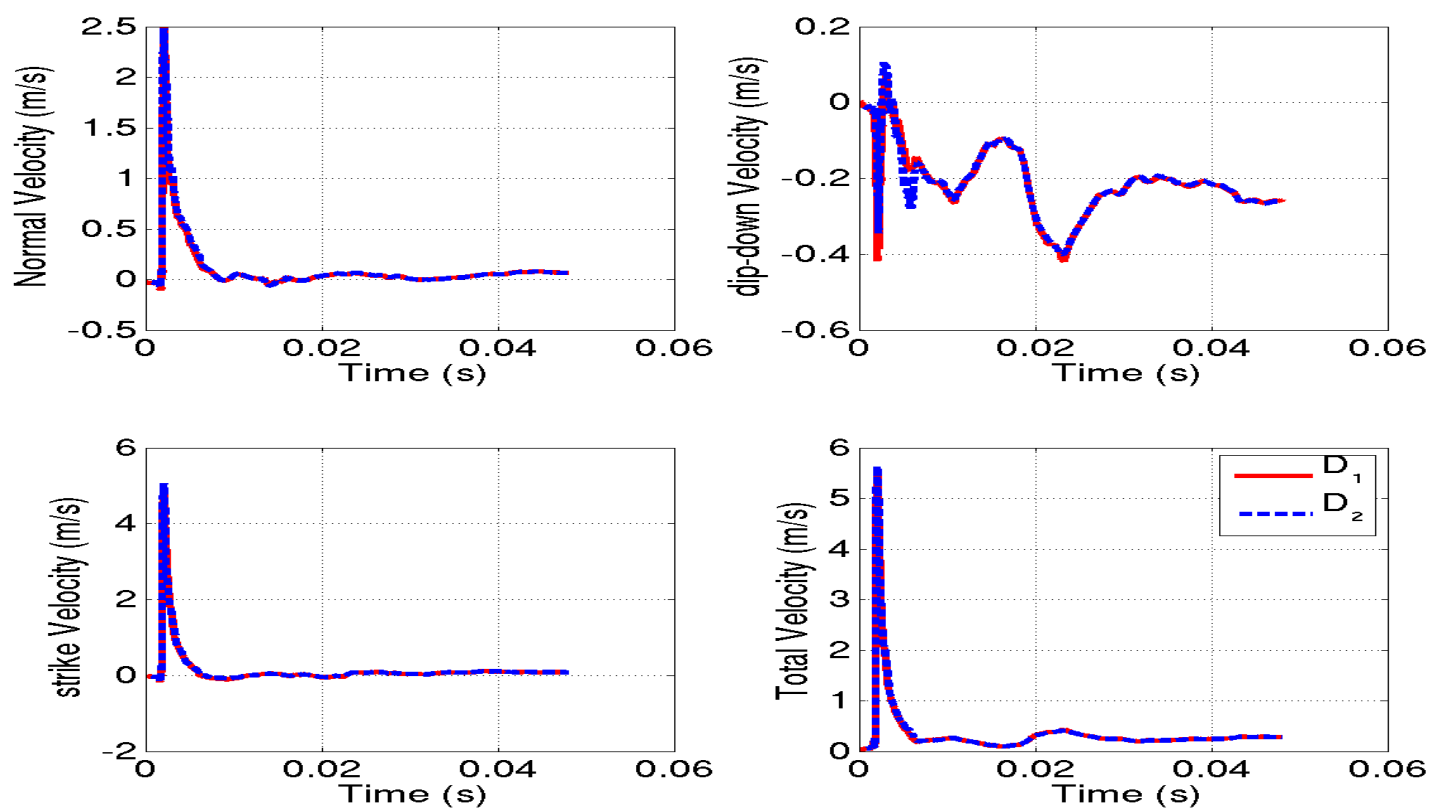

Figure 26. Comparison of wavefields across the faults planes at locations D1 and D2. Velocity components normal to the fault, along the strike and dip directions are shown. The results obtained shown are obtained from simulations where the level set method is used to model the faults surface.

\section{CONCLUSions}

We have performed analysis and simulations of SPE1-SPE3 events using both an Eulerian and a Lagrangian hydrocodes (GEODYN and GEODYN-L). Both codes agree with each other when the same material model is used.

We have used both an ensemble model, calibrated to match records from past nuclear events, as well as a new granite model calibrated for granite samples from the site, where SPE tests were conducted. The new model contains scalable parameters which allow us to apply it both to small and the large scales. Once scaled, it produces results similar to ones obtained with the ensemble model. In contrast to the ensemble model, the new model includes effects of poroelasticity and water saturation.

We have conducted discrete modeling of the SPE events with various joints properties to investigate the effects of joints on the wave anisotropy. It was shown that persistent joints sets can introduce anisotropy in the wave generation and cause significant tangential motions. 
We have used the nonlinear GEODYN code to predict the near field motions for the SPE3 shot which is designed to place in the same location as SPE2 and to test the effect of the damage zone. Both 2D axisymmetric (no faults) and fully three-dimensional simulations were performed. The damage zones sustained by SPE2 are retained as the initial condition for the SPE3 calculations. The results indicate that the peaks of the waveforms seem to decay slightly by the SPE2 damage however the pulse widths are reduced, indicating the energy is dissipated more by the damage zone as expected. Again we see the effects of the two faults exert relatively insignificant effects on the simulated wave motion response, that is, the non-spherical components are relatively small and the dominant motions are spherically symmetric.

Simulations of repeatable events (SPE2 and SPE3) with ensemble granite model showed that, the second explosion produces shorter pulse and has earlier arrival time. This is because the material is compacted during the first explosion. Since the ensemble model does not include any softening mechanism, this is a natural outcome. More simulations are underway where the damage is considered along with compaction.

\section{REFERENCES}

Antoun, T.H., Lomov, I.N., and Glenn, L.A. (2001), Development and Application of a Strength and Damage Model for Rock under Dynamic Loading, Proceedings of the 38th U.S. Rock Mechanics Symposium, Rock Mechanics in the National Interest, Edited by D. Elsworth, J. Tinucci, and K. Heasley, A.A. Balkema Publishers, Lisse, The Netherlands, 369-374.

Antoun, T.H, H. Xu, O. Vorobiev and I. Lomov, Initial near field predictions of the first two source physics experiments (SPE), LLNL report, 2011

Bandis, S.C., Lumsden, A.C.,Barton, N.R. (1983). Fundamentals of Rock Joint Deformation. Int. J. Rock.Mech.Min. Sci. Geomech. Abstr. 20(6),249-268

Barton,N., (1973), Engineering Geology 7, 287-332.

Barton, N. and Choubey, V. (1977), Rock Mechanics Felsmechanik Mecanique Des Roches 10, 1-54.

Broome, S., and T. Pfeifle (2011), "Phase 1 Mechanical Testing Results on Core from Borehole U-15n, Nevada National Security Site, in Support of NCNS Source Physics Experiment,” Technical Memo 6914, Sandia National Laboratories, June 8

Burchard, P., L-T Cheng, B. Merriman and S. Osher, "Motion of curves in three spatial dimensions using a level set approach", J. Computational Physics, 170, 720-742, 2001

Coulson, J.H. (1971), in Proceedings Of the 13th Symposium On Rock Mechanics, edited by E.J. Cording (Urbana, Ill, 1971), pp. 77-105.

Fossum, A.F., (1985). Effective Elastic Properties for a Randomly Jointed Rock Mass. Int. J. Rock. Mech. Min. Sci. Geomech. Abstr. 226,467-470

Glenn, L.A. and Goldstein, P. (1994), Journal of Geophysical Research, 99 (B6), 11723-11730. 
Lee,Y., Carr,J., Barr,D., and Haas, C. (1990), International Journal Of Rock Mechanics and Mining Sciences \& Geomechanics Abstracts 27, 453-464.

Lomov I.N., Antoun T. and Vorobiev O.Yu "Comparison of Joint Modeling Approaches Including Eulerian Sliding Interfaces”, LLNL-TR-421580, 2009

Schock, R. N., Heard, H. C., and Stephens, D. R., 1973, "Stress-Strain Behavior of a Granodiorite and Two Craywackes on Compression to 20 Kilobars," J. Geophys. Res., Vol.78(36), pp. 5922-5941.

Rubin, M.B, Vorobiev, O.Yu., Glenn, L.A., 2000, "Mechanical and numerical modeling of a porous elastic-viscoplastic material with tensile failure," Int. J. Solids and Structure, Vol.37, pp.1841-1871.

Tatone, B.A. and Grasselli, G. (2010), International Journal of Rock Mechanics and Mining Sciences 47, 1391-1400

Vorobiev, O. (2008). Generic strength model for dry jointed rock masses. International Journal of Plasticity, 24(12), 2221-2247.

Vorobiev O.Yu and Antoun T., "Equivalent continuum modeling for nonlinear wave propagation in jointed media", Int.J.Numer.Meth.Engng, 2011,pp. 1101-1124

Vorobiev, O. (2011) Simple Common Plane Contact Algorithm, Int.Journ.Num.Meth.Engng,243-268. 


\title{
FAR-FIELD MODELING
}

\author{
R. J. Mellors, A. Pitarka, A. J. Rodgers, W. R. Walter, S. Ford, H. Xu, E. Matzel, S. Myers, N. \\ A. PETERSSON, B. SJOGREEN, T. HAUK, AND J. WAGONER
}

\section{INTRODUCTION}

The Source Physics Experiment (SPE) is a research effort aimed at improving the modeling and simulation of explosions by developing a better physical understanding of the fundamental controls on wave generation and propagation. A particular focus is on shear waves, which are poorly predicted by existing explosion models, despite their important role in event identification and yield estimation. The SPE is intended as a series of chemical explosions (Table 1), of varying sizes and settings, which will be recorded on extensive instrumentation located both near the source and at extended distances. One objective is to predict the waveforms prior to each shot as a test of the planned gradual improvement of predictive capability of our source and path models and codes. This report is intended to serve as a summary of the pre-shot simulation of the 'far-field' effects of the July 24, 2012 shot (SPE3). By 'far-field' we refer to distances at which seismic wave propagation is primarily elastic [Rodgers et al., 2010].

Table 1. SPE shot parameters

\begin{tabular}{|l|l|l|l|l|l|}
\hline & TNT equiv. (kg) & $\begin{array}{l}\text { SHANFO } \\
(\mathbf{k g})\end{array}$ & $\begin{array}{l}\text { Depth } \\
(\boldsymbol{m})\end{array}$ & Comments & $\begin{array}{l}\text { Date/Time } \\
(\boldsymbol{G M T})\end{array}$ \\
\hline SPE1 & 100 & & 60 & Calibration & $05 / 03 / 11$ \\
\hline SPE2 & 1000 & 1166 & 45 & & $10 / 25 / 11$ \\
\hline SPE3 & 1000 & 1166 & 45 & Damage zone & nominal \\
\hline
\end{tabular}

This report summarizes some of the results from the data analysis from SPE1 and SPE2 [Mellors et al., $2011 \mathrm{a}, \mathrm{b}]$, compares the results with the pre-shot simulations, and then documents improvements in the modeling and numerical capabilities as a result of the previous analysis along with new simulations and 
predictions for the SPE3 dataset. Improvements include a more complex source model based on 3D near-field nonlinear modeling, a refined velocity model, and estimates of the expected source differences.

Geologic setting. The SPE shots take place in the northeast corner of the Nevada National Security Site (NNSS) (Figure 1). The NNSS possesses a complex geologic setting with strong lateral and vertical variations in geophysical properties relevant to seismic wave propagation. The SPE1 ground zero is located in an outcrop of granite surrounded by volcanic and sedimentary rocks. The main units near the SPE site consist of the granite (Climax Stock), alluvium, Tertiary volcanic tuffs, and Pre-Tertiary sedimentary rocks (mainly carbonates). The alluvium, which is relatively low-density is thickest southwest of the shot point, in the topographically flat Yucca Flat. Yucca Flat is also notable for a deep water table, which reduces shallow seismic velocities. Northwest of the shot point the topography increases with a steep gradient and the area is dominated by pre-Tertiary carbonates. For a detailed description of NNSS geology see Howard [1985] and for the Climax Stock/ Yucca Flat area see GSG, [2006].

SPE seismic network. A variety of instruments including short-period geophones (GS11d, Omni 2400), accelerometers (Episensors), rotational sensors (eentec R1) and broad-band (CMG40, Trilliums, and PMD) have been deployed (Figure 1). Most of the instruments were installed in 5 radial lines centered on the shot location, with the closest instruments spaced $100 \mathrm{~m}$ away. Spacing between sites was $100 \mathrm{~m}$ up to a distance of $2 \mathrm{~km}$ from the shot point. Lines 3, 4 and 5 extended further with broadband instruments located out to a distance of $10 \mathrm{~km}$. Nine other sites not located on the radial lines were also occupied by

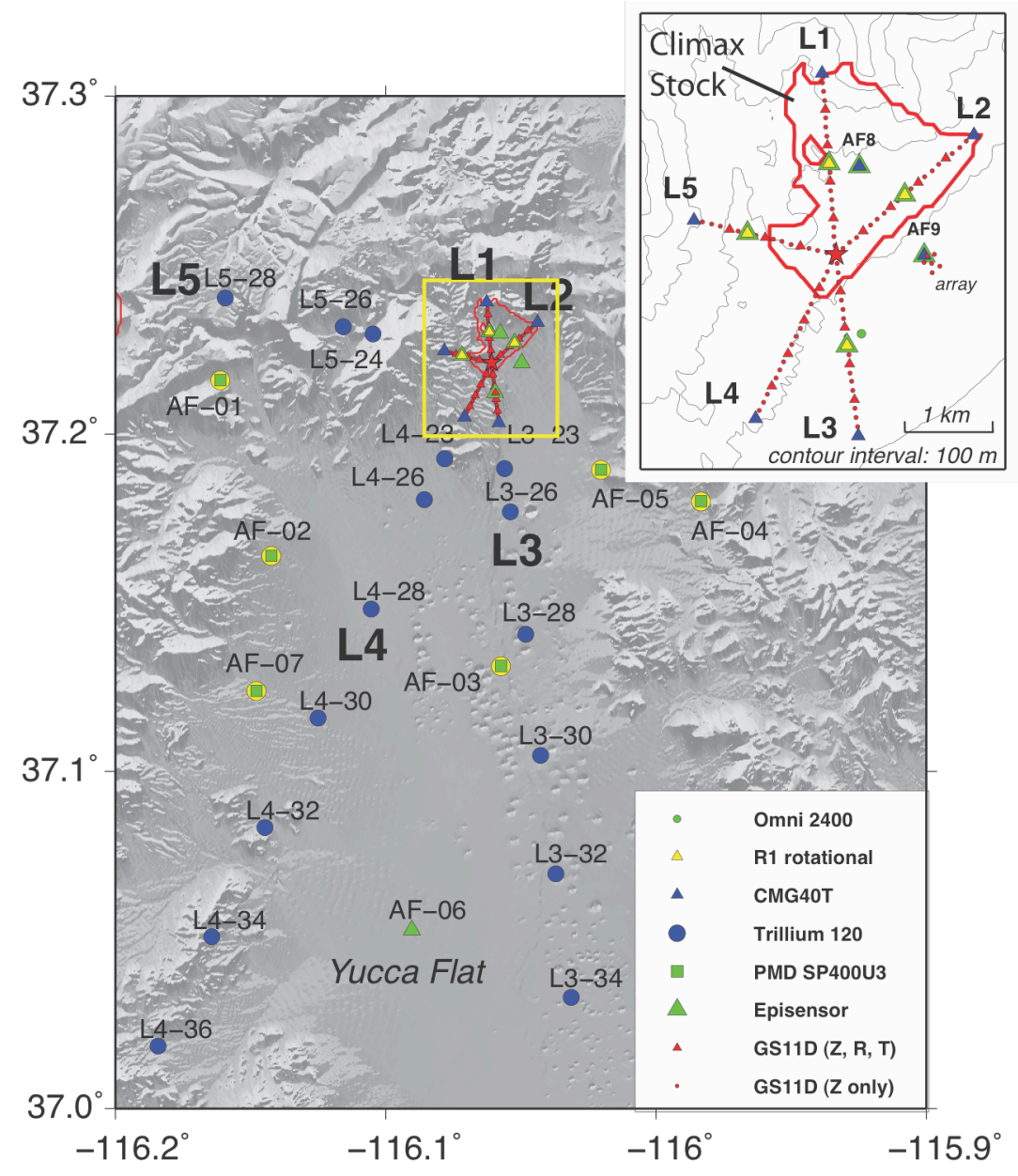

Figure 1. Far-field seismic sensor deployment for SPE3. 
either broadband or accelerometers. A small array of five geophones was installed after SPE2 to the southeast. Data is also available from the University of Reno seismic network. Instruments are located on concrete pads set in the ground. Instruments within $2 \mathrm{~km}$ of the shot point are installed with horizontal components oriented radially and tangentially towards the shot point and instruments farther than $2 \mathrm{~km}$ are oriented with respect to cardinal direction (N-S, E-W, and Z). Recording for most instruments are on Reftek digitizers at 250 or $500 \mathrm{~Hz}$. Selected site are recorded using radio telemetry by the UNR network.

\section{MODELING APPROACHES AND PREVIOUS SIMULATIONS}

As mentioned above, both purely numerical and semi-empirical techniques were applied to model the SPE shots. The numerical techniques applied a 3D finite difference algorithm using a 3D velocity model while the semi-empirical techniques scaled previous waveforms using source approximations. 3D finite difference has the advantage of flexibility but is limited in frequency range, as the higher frequencies are computationally intractable for problems of this size. Semi-empirical approaches do not have frequency limits but include a priori assumptions. Prior to the SPE1 shot, a comprehensive numerical modeling effort was undertaken and presented in Rodgers et al. [2010]. The modeling used an advanced finite-difference algorithm (WPP) [Petersson and Sjogreen, 2009, 2010, 2011; Sjogreen and Petersson, 2010] to conduct 3D modeling of the seismic waveforms at distance up to $4 \mathrm{~km}$ from the source. WPP has the capability to handle $3 \mathrm{D}$ variations in the material properties (density, compressional and shear wavespeed and the attenuation factor, Q) and a variety of sources including point sources and extended sources using the moment tensor representation. The free-surface boundary can include topography and the remaining sides of the domain include absorbing boundaries. It also includes depth-dependent mesh refinement to improve performance for models with increased wavespeeds at depth [Petersson, 2011]. WPP simulations can include two types of source parameterizations: an isotropic source embedded in WPP and a coupled nonlinear source, calculated by a hydrodynamic code (GEODYN), which is coupled (one-way) to WPP [Rodgers et al., 2011].

GEODYN is a 3D Eulerian hydrocode [Antoun et al., 2001, 2004, 2011; Antoun and Lomov, 2003; Lomov et al., 2003], which simulates the hydrodynamic response of materials to explosion loading and includes the capability to handle nonlinear effects such as tensile failure and yielding [Rodgers et al, 2011]. A Jones-Wilkins-Lee (JWL) equation of state is used to estimate the response of the ANFO explosive mix in the SPE1 and SPE2 shots. Velocities within a $100 \mathrm{~m}$ cube are generated and relayed to WPP as a driving boundary condition. The advantage of linking GEODYN with WPP is that GEODYN handles the source region accurately but is numerically expensive; WPP can extend the motions in the elastic regime where most seismic measurements are made.

Coupling the two codes has been challenging and therefore simple source models are being tested initially, as these are easier to link with WPP and are useful for comparison to other models, such as isotropic or CLVD sources. The initial GEODYN model used for SPE2 was axisymmetric and hence cannot generate transverse motions. A full 3D GEODYN source, which should be capable of generating transverse motions, was tested for SPE3. 
Far-field velocity model. A 3D model (Figure 2) was constructed using a set of four layers (alluvium, Tertiary, Pre-Tertiary, and granite) to represent the complex geologic setting of the SPE test site. Interfaces between layers were imported from a GIS-based geologic model of the area and based mainly on surface mapping, borehole data, and geophysical modeling. Layer depths between known points were defined by interpolation within the GIS program (EarthVision) and then reformatted to match WPP input format. Each layer interface varied laterally and homogenous material properties were assigned to each layer based on previous studies. This model is the basis for all following improved models.

Based on this geologic representation, a set of four simulations for the SPE1 shot were conducted using a domain of $8 \mathrm{~km}$ by $8 \mathrm{~km}$ by $5 \mathrm{~km}$ with a grid spacing of 5 meters. These models increased in complexity and included: 1) homogenous half-space with a flat boundary; 2)

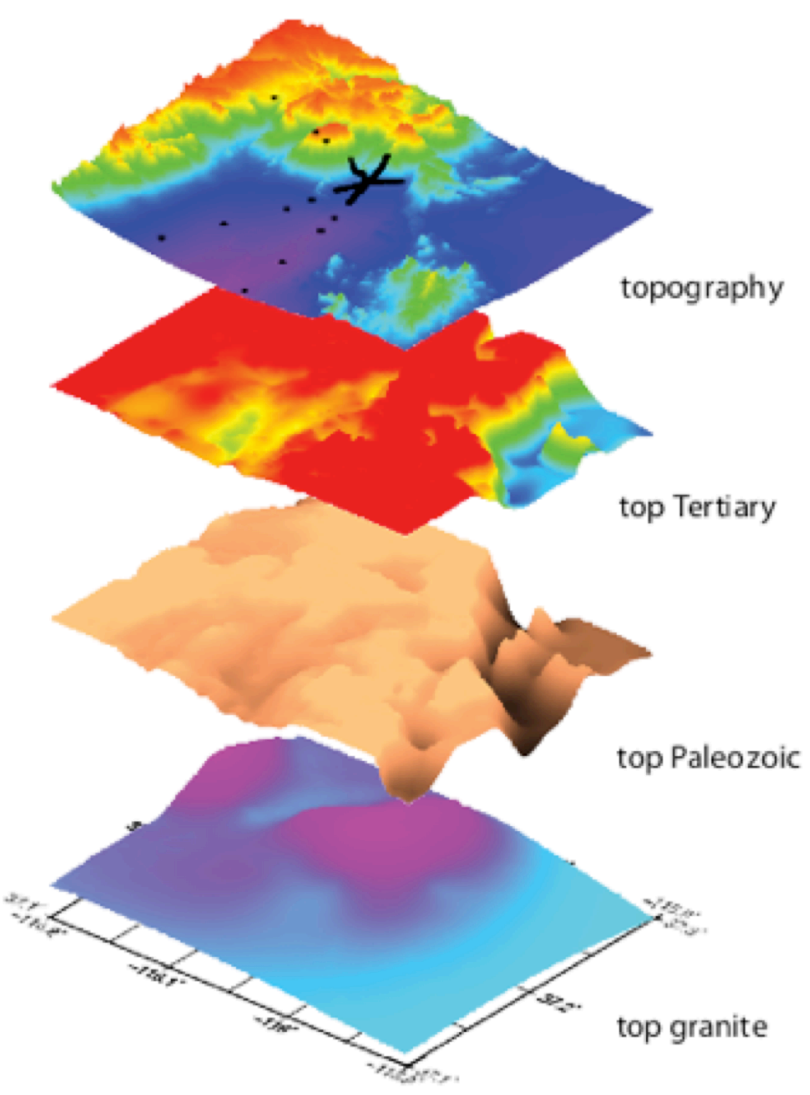

Figure 2. Perspective view of layers and boundaries. homogenous half-space with topography; 3) 3D heterogeneity with a flat boundary; and 4) 3D heterogeneity with topography. The results indicated that the $3 \mathrm{D}$ volumetric structure would have a pronounced effect on the ground motions, especially to the southwest as the wavefield propagated into the low velocity alluvium of Yucca Flat, where high amplitudes were generated. The effect was expected to be strongest on Lines 3 and 4. Volume scattering from heterogeneities in the velocity model caused scattering, which appears as transverse energy. This is most evident on lines 3 and 4 . Some scattering due to topography was expected to the northwest (Figure 3). 
SPE1. The SPE1 shot was a chemical explosion of $100 \mathrm{~kg}$ (TNT equivalent) of explosives detonated at a depth of $60 \mathrm{~m}$ on May 3, 2011. A comprehensive data set was collected (Figures 4 and 5) and data recovery was good, with approximately $95 \%$ return of useful data. Some anomalies were noted in recorded amplitudes for selected stations, possibly due to instrument or cable problems [Mellors et al., 2011]. Clear differences along and between lines were observed, which in general matched the predictions. Lines 3 and 4 were delayed relative to the other lines and strong variations in amplitude and waveforms were observed, with pronounced surface waves visible on Lines 3 and 4 . The synthetics underestimated the relative proportion of transverse energy on Lines 1 and 2. At this distance, the seismic waves traveled only through the granite (Climax Stock) for Lines 1 and 2. As it was modeled as a homogenous media, little scattering was expected. The amount of transverse energy observed in the data

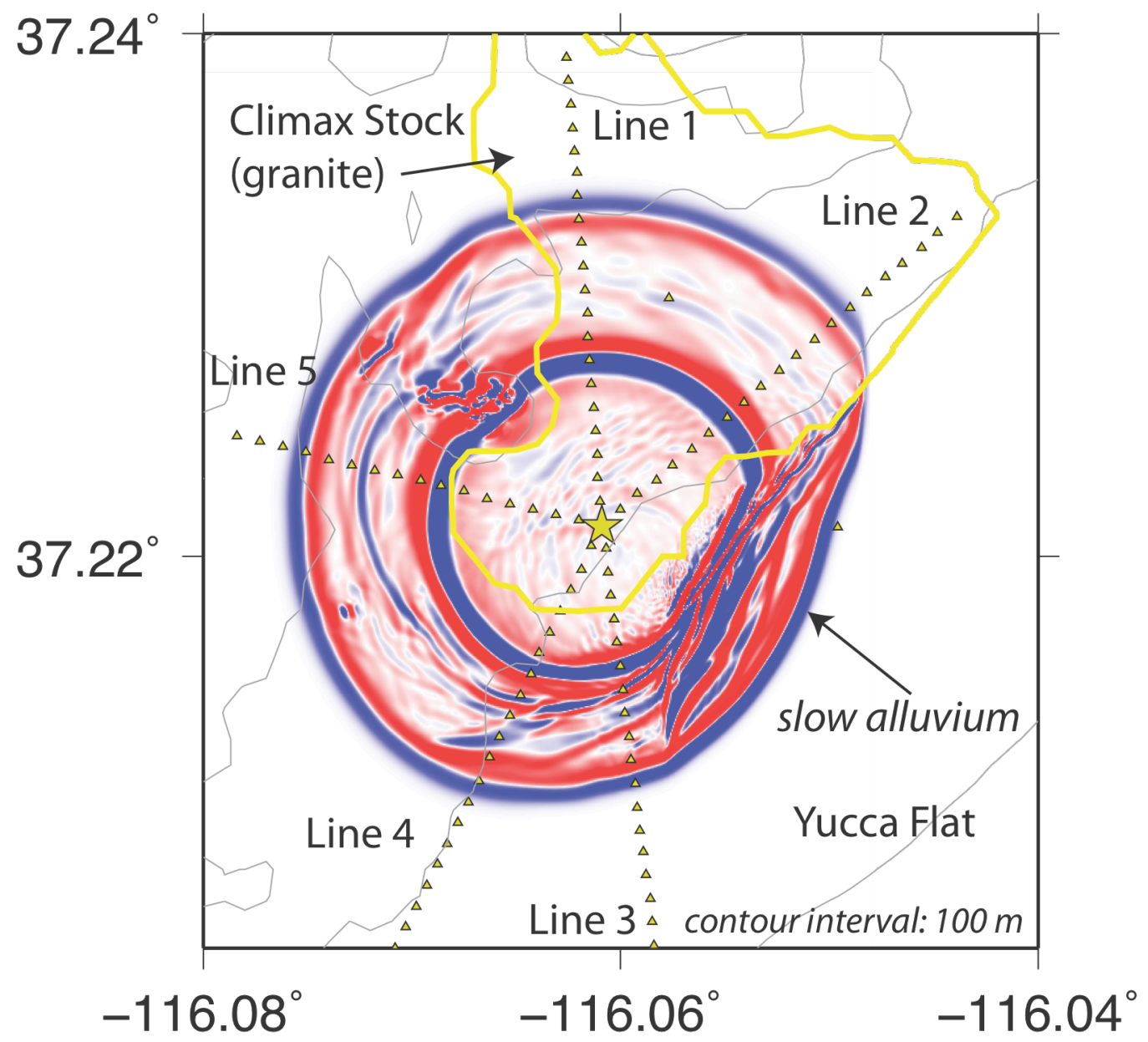

Figure 3. Example snapshot of the SPE simulated wavefield using WPP program and a $3 D$ velocity model with surface topography. 
suggests that either the source generated the transverse energy or an unexpected amount of scattering occurred within the granite. Topographic effects were predicted but these were difficult to distinguish in the data from 3D volume effects and also possibly because the stations with expected pronounced topographic effect were not deployed along line 5 due to difficulty of access.

Systematic differences in travel times between the Version 1 model and the data were observed. Lines 3 ,
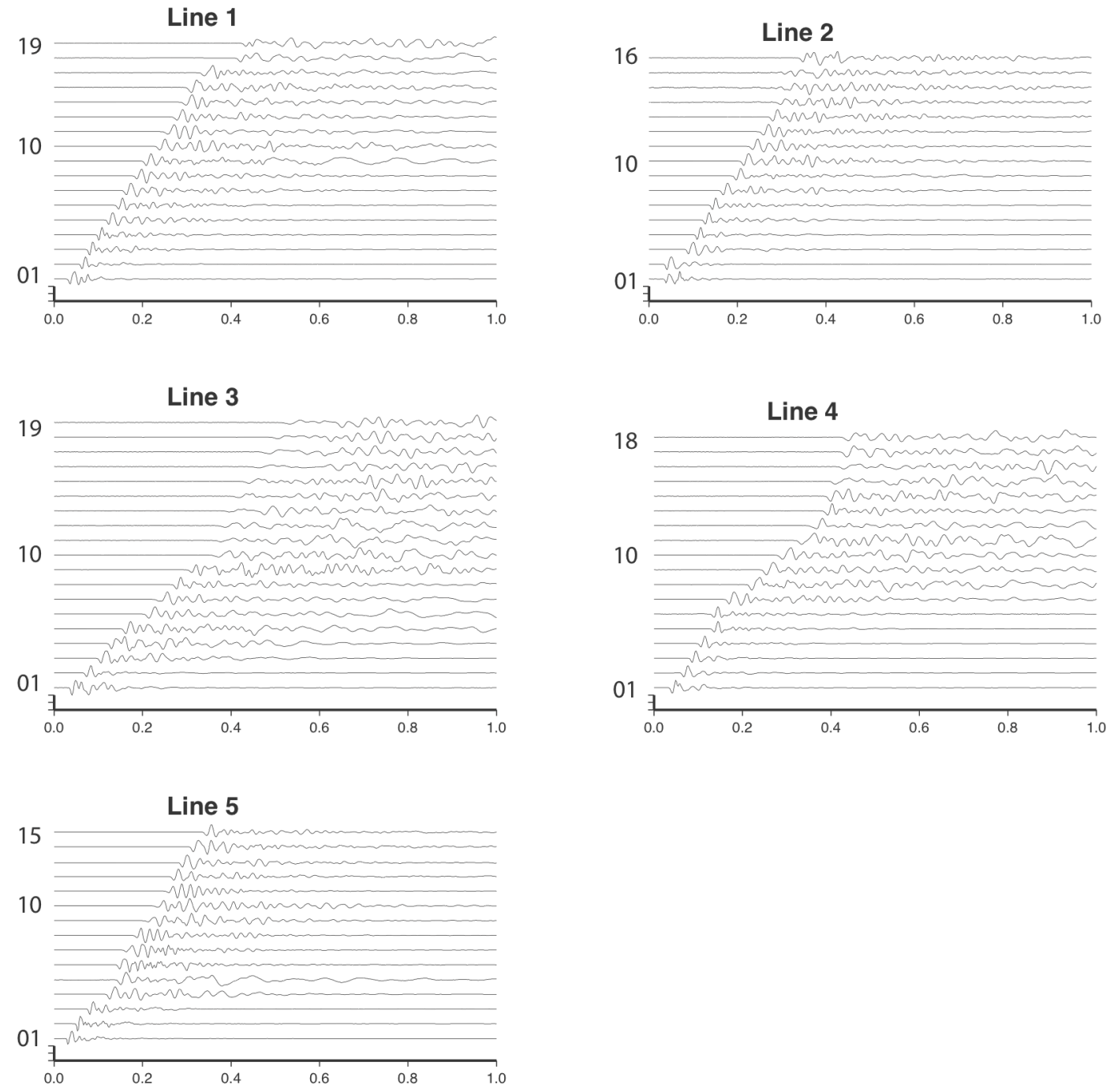

Figure 4. Seismic data (normalized, vertical component) recorded from the SPE1 shot showing stations numbers at left. Traces with high noise or instrumental problems have been omitted. Lines 1 and 2 are almost entirely within the granite while only first few stations of Lines 3 and 4 are on granite before transitioning to alluvium or Tertiary tuffs. Note changes in first arrival moveout and amplitude at a distance of approximately $1 \mathrm{~km}$ on Lines 3 and 4. Line 5 extends from granite to Pre-Tertiary. 
4 and 5 show indication of a change in slope in the plot of time versus distance at distances of roughly 1 $\mathrm{km}$ and this may reflect a refracted wave as well as changes in the uppermost layer velocity. As a result of these observations, the velocities in the model were altered to match the observed data.

In parallel with this effort ambient noise cross-correlation was conducted to develop empirical Green's functions of the interstation velocity structure. This appears to be a promising approach to refining the velocity model although the low gain setting on the geophones has increased the ratio of instrumental
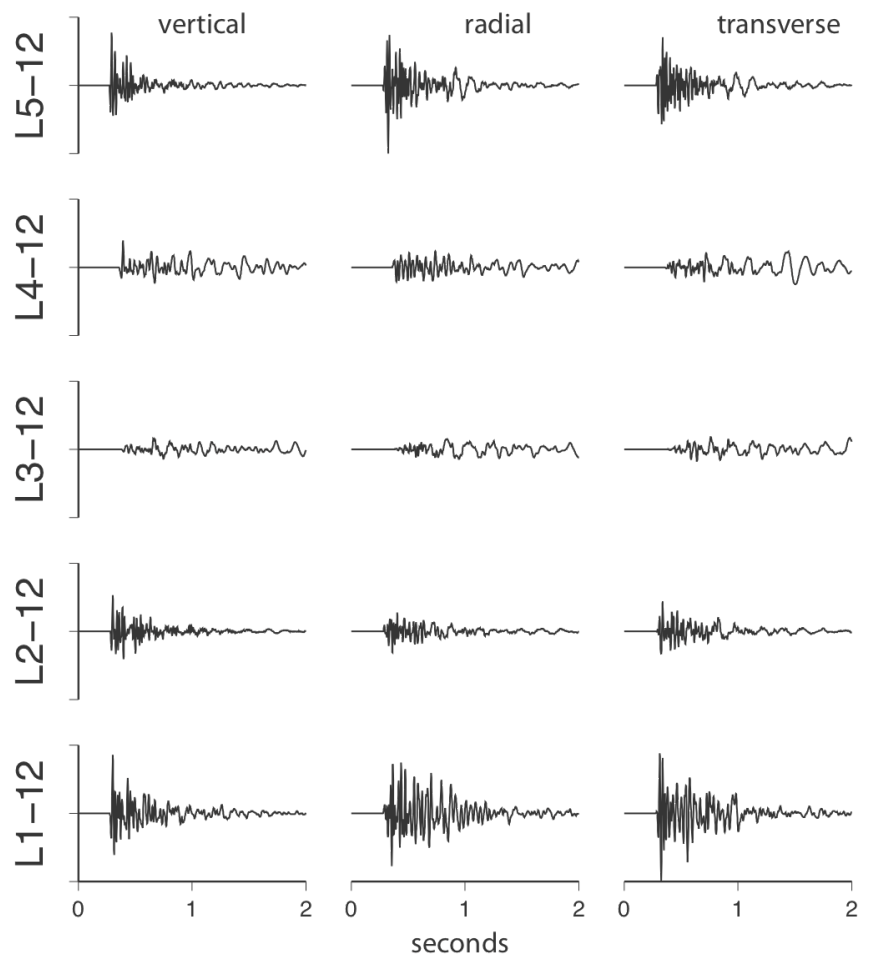

Figure 5. Vertical, radial, and transverse components of seismograms recorded at a distance of $1.2 \mathrm{~km}$ from the shot. Labels on the vertical axis refer to the line (L1, L2, L3, L4 and L5) and the station (12).

The $L 3$ and $L 4$ lines show a delayed arrival and lower frequencies, as predicted.

noise to seismic noise. At short distances (less than $300 \mathrm{~m}$ ) the noise is highly correlated at the frequencies recorded by the GS11d geophones but decreases rapidly with distance. The gain on the geophones has been adjusted to record high gain (gain=32) data for several weeks to allow seismic interferometry.

Using the revised model, the fit between predicted and observed (SPE1) waveforms was examined. The predicted traveltimes were fit reasonably well but waveform fits were poor. An approximate match was observed for filtered (bandpass $2-5 \mathrm{~Hz}$ ) for lines 1 and 2 but significantly poorer for lines 3-5. The observed data showed generation of surface waves at distances greater than $0.5 \mathrm{~km}$ along line 4 which were not generated in the synthetics. This is likely due to the artificially high $(1000 \mathrm{~m} / \mathrm{s})$ shear wave 
velocity in the model as compared to the near surface velocities in Yucca Flat, which may be as low as $500 \mathrm{~m} / \mathrm{s}$ based on borehole seismic velocity measurements [Howard, 1985].

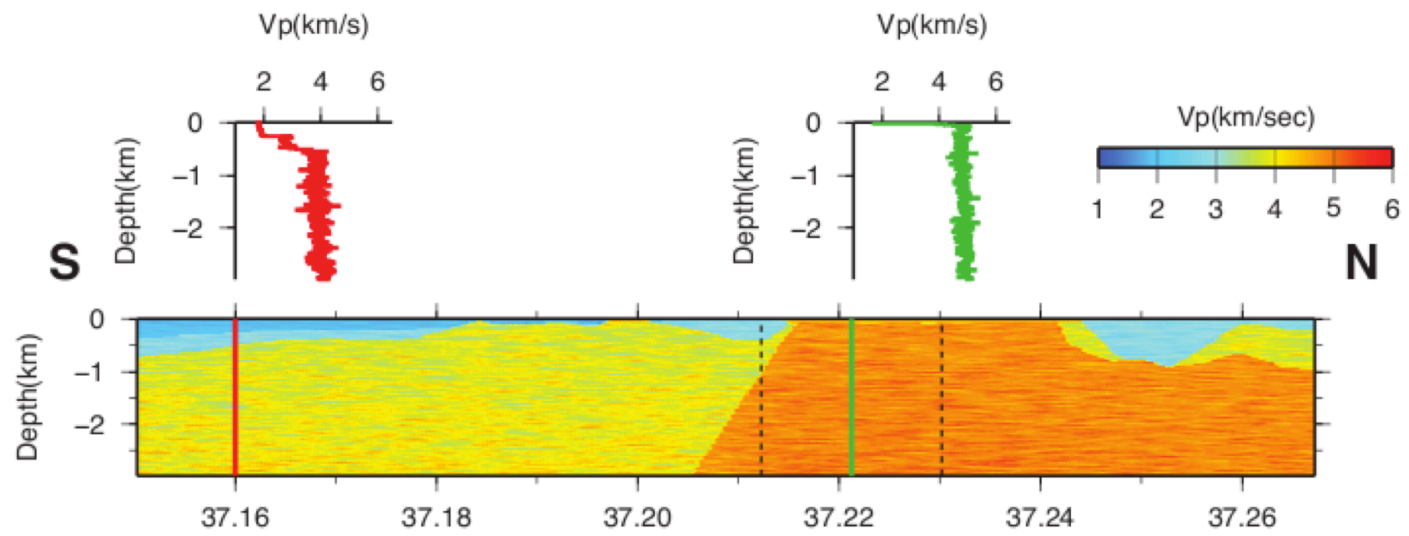

Figure 6. $N$-S cross-section of the $3 D$ velocity model through the SPE site. Top panels show Vp as a function of depth at Yucca Flat (red line) and SPE site (green line).

SPE2 simulations. SPE2. The SPE2 shot was a chemical explosion of $1000 \mathrm{~kg}$ (TNT equivalent) at a depth of $45 \mathrm{~m}$ on October 25, 2011 in the same borehole as SPE1. The same seismic network was in place for the SPE2 shot and a set of simulations was conducted for the SPE2. These simulations use the revised model and model the SPE2 waveforms for standard orthogonal motions (x,y, and $\mathrm{z}$ ) and also for rotational motion.

For SPE2, both pure finite difference and a GEODYN source (axial symmetric) were tested. This was the first use of the GEODYN source in a WPP simulation. Both Cartesian and rotational waveforms were modeled. Results were encouraging but again failed to replicate the amplitude of the transverse energy. However, semi-empirical approaches, which used the SPE1 waveforms as a template scaled by the expected Mueller-Murphy source spectra, did predict the observed waveforms well both in phase and amplitude. The SPE2 waveform was very similar to the SPE1 waveforms but an order of magnitude larger in amplitude. 


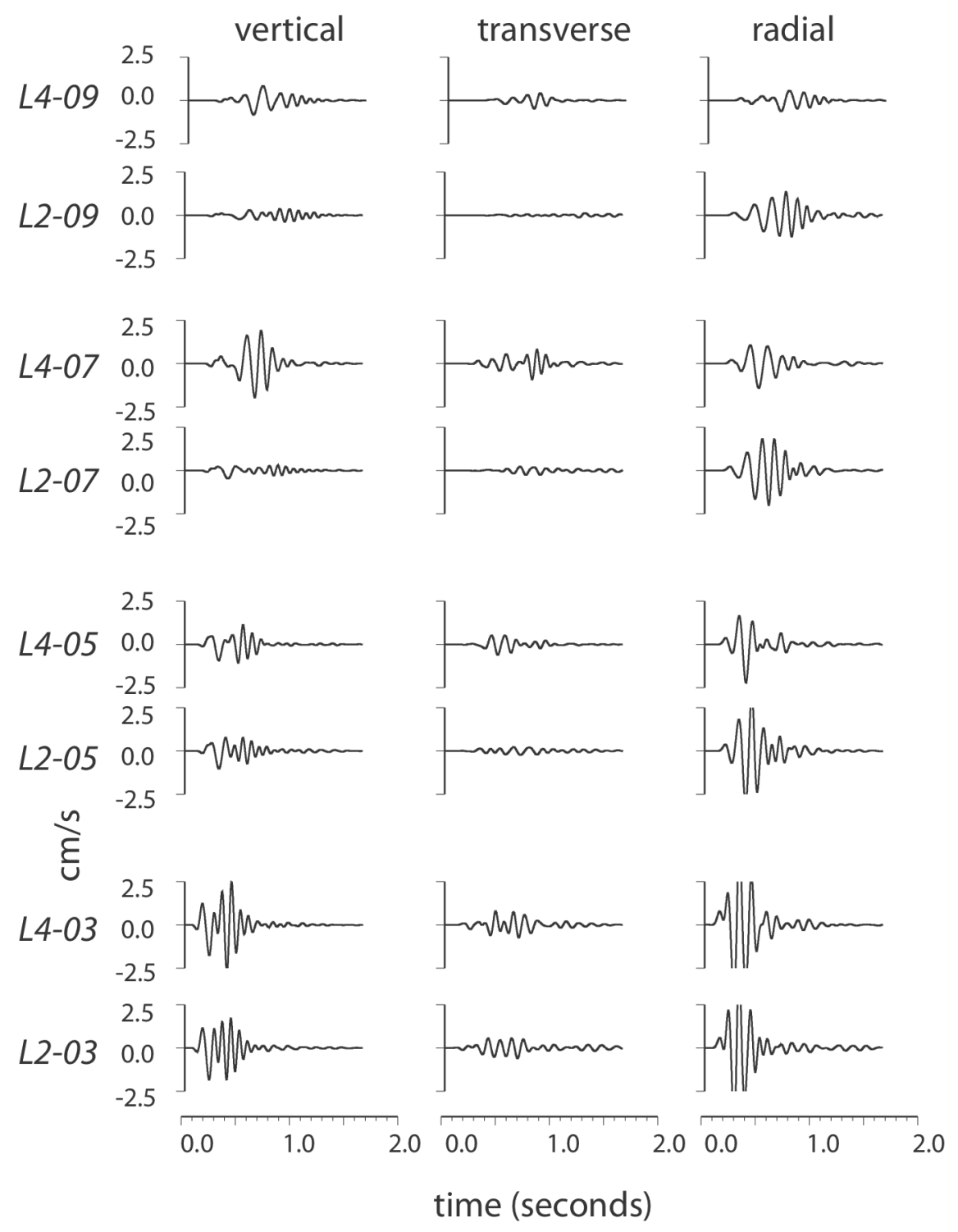

Figure 7. Synthetic seismograms of expected motion using an isotropic explosion source for selected stations at distance of less than $1 \mathrm{~km}$ on lines 2 and 4 . The addition of a shallow low velocity zone and heterogeneities produce significant tangential energy.

\section{SPE3 SIMULATIONS}

For SPE3, the challenges are twofold: 1) improve the overall velocity model to match the previous simulations and 2) compensate for the effect of the damage zone caused by the SPE2 shot in the granite. Progress was made in both areas and the revisions to the velocity model improved the fit to the previous data and especially on the tangential components. The 3D velocity model was further revised. First, a low velocity layer underlain by a gradient was added to the granite model. The change was based on refraction 
tomography [Abbott et al., 2011] and by 2.5 D finite difference modeling of SPE2 waveforms recorded along the L1 and L2 profiles. Second, based on velocity variations measured from well logs, random heterogeneities with a scale length of $350 \mathrm{~m}$ in the horizontal and $40 \mathrm{~m}$ in the vertical and an amplitude of $15 \%$ were added to the model layers to enhance scattering (Figure 6). A node spacing of $5 \mathrm{~m}$ with an approximate maximum frequency of $10 \mathrm{~Hz}$ was used. This greatly improved the fit of the waveforms and also increased the sensitivity to topographic effects within the granite. Figure 7 shows example seismograms using an isotropic Mueller-Murphy source in this model.

As the primary objective of SPE2 is to evaluate the effect of a damage zone on a repeated shot, this was the focus of considerable effort. Mueller

and Murphy [1971] developed a relationship to characterize source scaling from empirical and theoretical constraints that have been widely applied. A Mueller-Murphy model is a partly empirical model that includes a variable cavity radius, elastic radius, and material properties such as density and seismic velocities.

The Mueller-Murphy source was varied to mimic the effect of shattered rock immediately around the source (the damage zone). A major difficulty with this approach is the uncertainty regarding the expected variation in elastic parameters near the damage zone but outside the elastic radius. Therefore, we tested two possibilities: a damage zone

Table 2. Mueller-Murphy parameters for SPE1 and SPE2. A yield of 1.97e-03 was used.

\begin{tabular}{|l|l|l|l|l|l|l|}
\hline model & shot & $\mathbf{V p}$ & $\mathbf{V s}$ & Density & Material & Depth \\
& & $\mathbf{k m} / \mathbf{s}$ & $\mathbf{k m} / \mathbf{s}$ & $\mathbf{( k g / m 3 )}$ & & $\mathbf{( m )}$ \\
\hline \multirow{2}{*}{ A } & SPE2 & 4.8 & 2.7 & 2500 & granite & 45 \\
\cline { 2 - 7 } & SPE3 & 4.0 & 2.3 & 2300 & granite & 45 \\
\hline \multirow{2}{*}{ B } & SPE2 & 4.8 & 2.7 & 2500 & granite & 45 \\
\cline { 2 - 7 } & SPE2 & 4.8 & 2.7 & 2500 & weak & 45 \\
& & & & & granite & \\
\hline
\end{tabular}
with lower seismic velocities and densities and one with identical velocities but a weaker rock (Table 2). Figure 8 shows the expected Mueller-Murphy source-time functions for SPE2 and SPE3 compared in both time domain and frequency domain versions. Parameters correspond to the values in Table 2 and are for granite using values in Stevens and Day [1985].

Figures $9 a$ and $9 b$ compare the expected seismograms after using the source functions shown in Figure 8 to drive the 3D finite difference code. The revised 3D velocity model (e.g. Figure 6) with heterogeneities to cause scattering. For model A (9a) the SPE3 waveforms show slightly higher amplitudes than SPE2 due to the lower shear wave velocities at the source but for model B (Figure 9b), SPE3 shows lower amplitudes due to more energy absorption. While neither result was significantly different, these conflicting results illustrate the challenging nature of the problem. 
Figure 10 shows an empirical estimation of the SPE3 waveform by calculating a ratio (P-wave) between the two Mueller-Murphy spectra and convolving with the SPE2 waveform. As in the finite difference waveforms, the SPE3 amplitudes are slightly higher than the SPE2 amplitudes. A major difficulty with this approach is the uncertainty regarding the expected variation in elastic parameters near the damage zone but outside the elastic radius. Here we have assumed that seismic wave velocity and density has decreased, which implies a significant change in the Lame parameters. The near-field calculations, which included compaction effects caused by SPE2, suggested that shock wave velocities for SPE3 may be faster than SPE2. However, these calculations did not include any softening of the material. The Mueller-Murphy model does not allow for a specific change in cavity size (although this may be
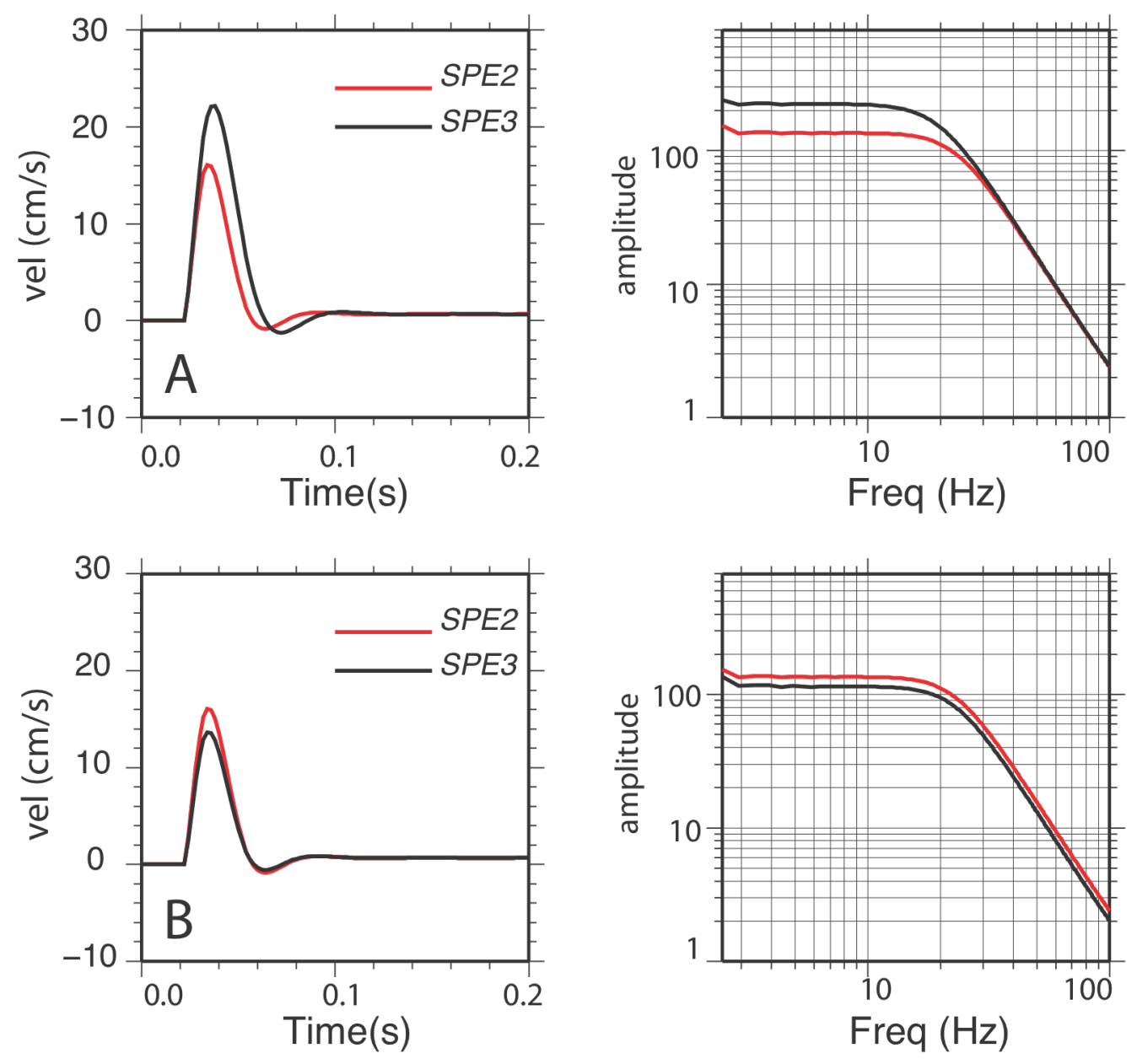

Figure 8. Mueller-Murphy source function (time on left, frequency on right) for SPE2 and SPE3 for two different representations of the source material. The upper set (A) assumes a lower velocity and density for SPE3 dues to a damage zone. The lower set (B) assumes that the damaged granite retains the same seismic velocities but is weaker and absorbs more energy. 
encompassed by a change in elastic radius) or a change in attenuation in the elastic regime. It is unclear how well these will impact the results.

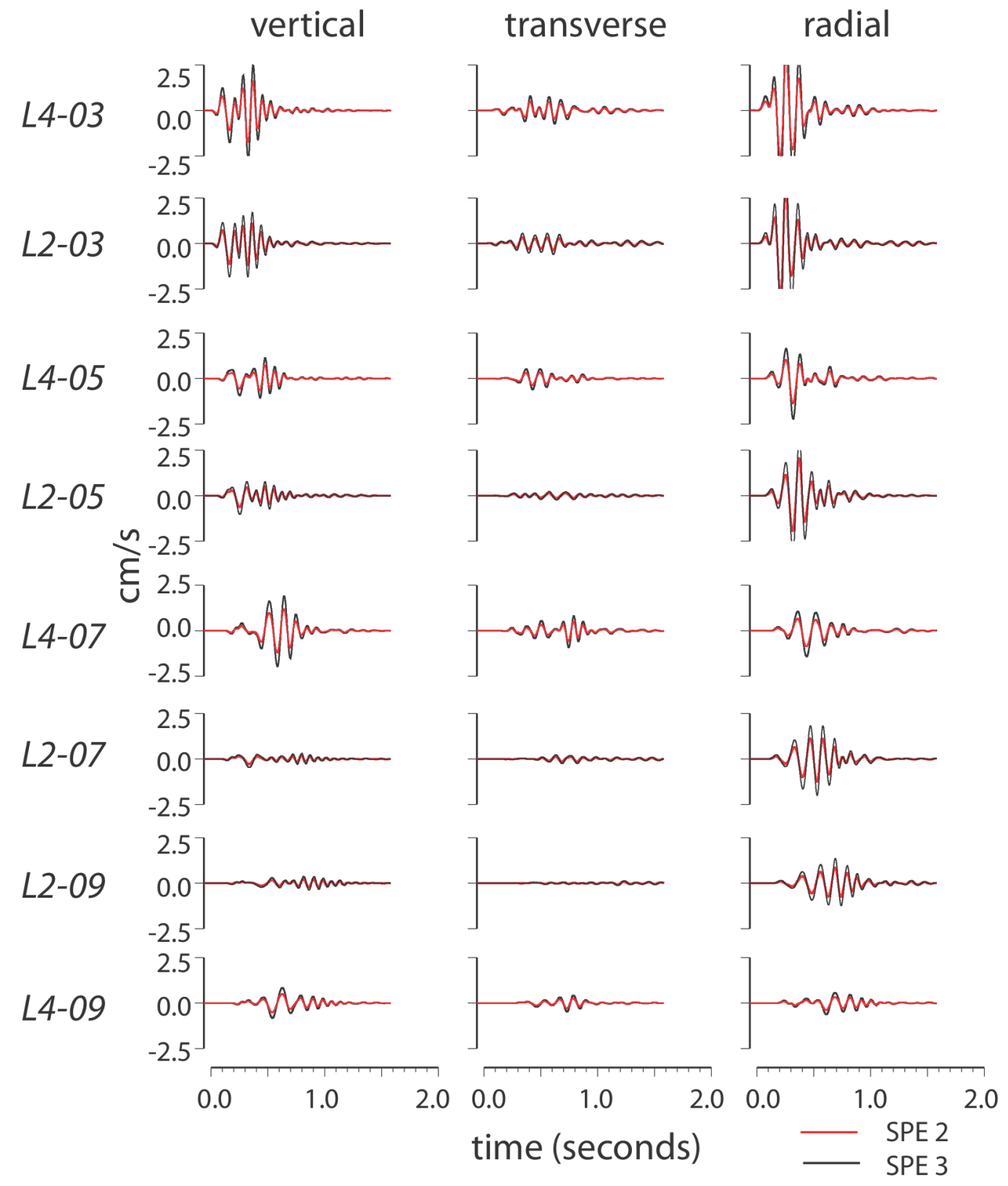

Figure 9a. Comparison of SPE2 and SPE3 for a damage zone with lower seismic velocities. 


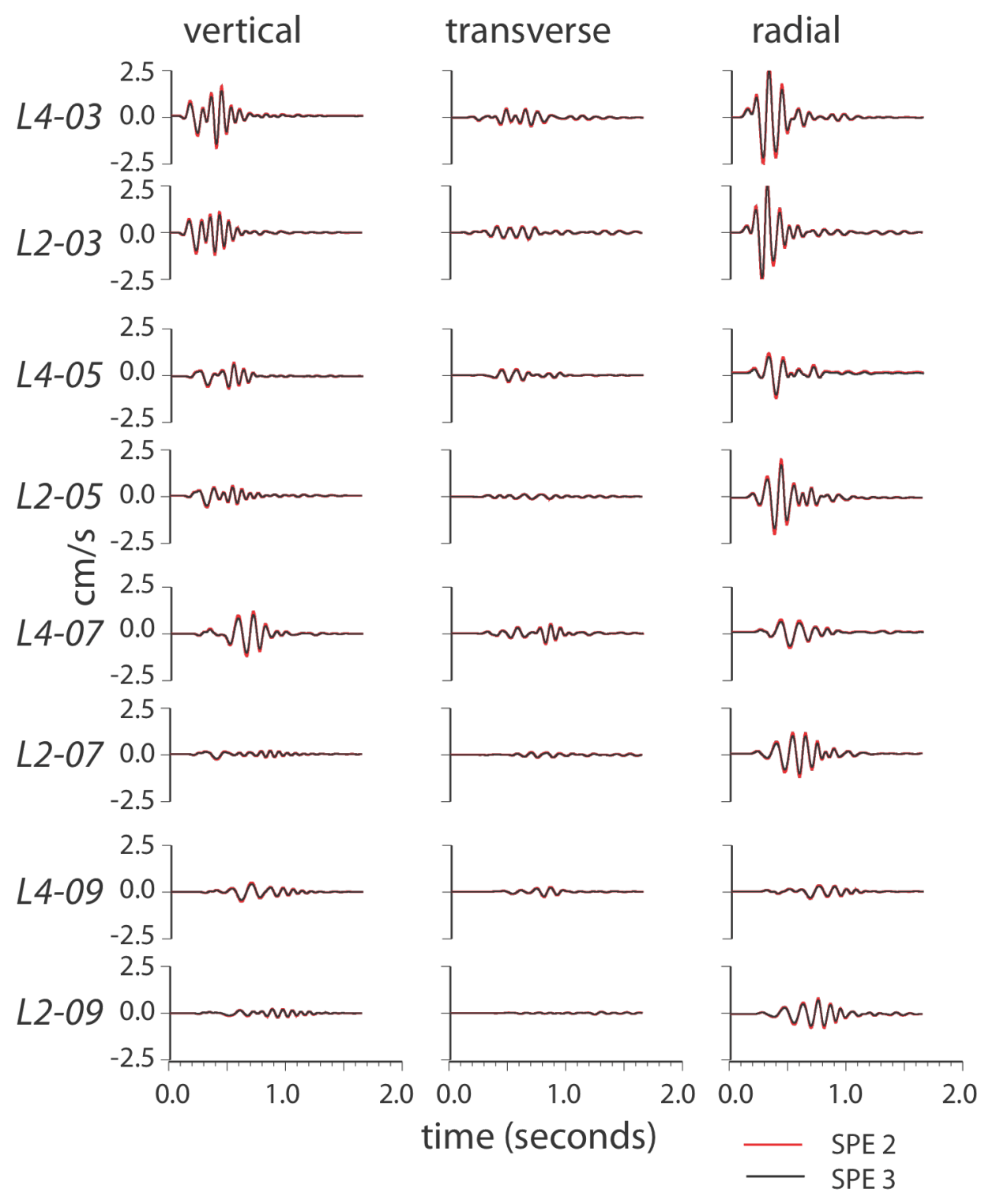

Figure 9b. Comparison of SPE2 (red) with SPE3 (black) for a damage zone with identical seismic velocities but weaker granite.

GEODYN/WPP direct coupling. We also tested coupling the WPP model with GEODYN in the full 3D case (Figure 11). As near-field motions are poorly constrained due to observational issues, we decided on focusing on increasing capabilities and validation rather than attempting computationally intensive full far-field predictions at this point. Note that the WPP source uses an a priori seismic moment as source size and does not specifically include any coupling factor. In contrast, the GEODYN model estimates 


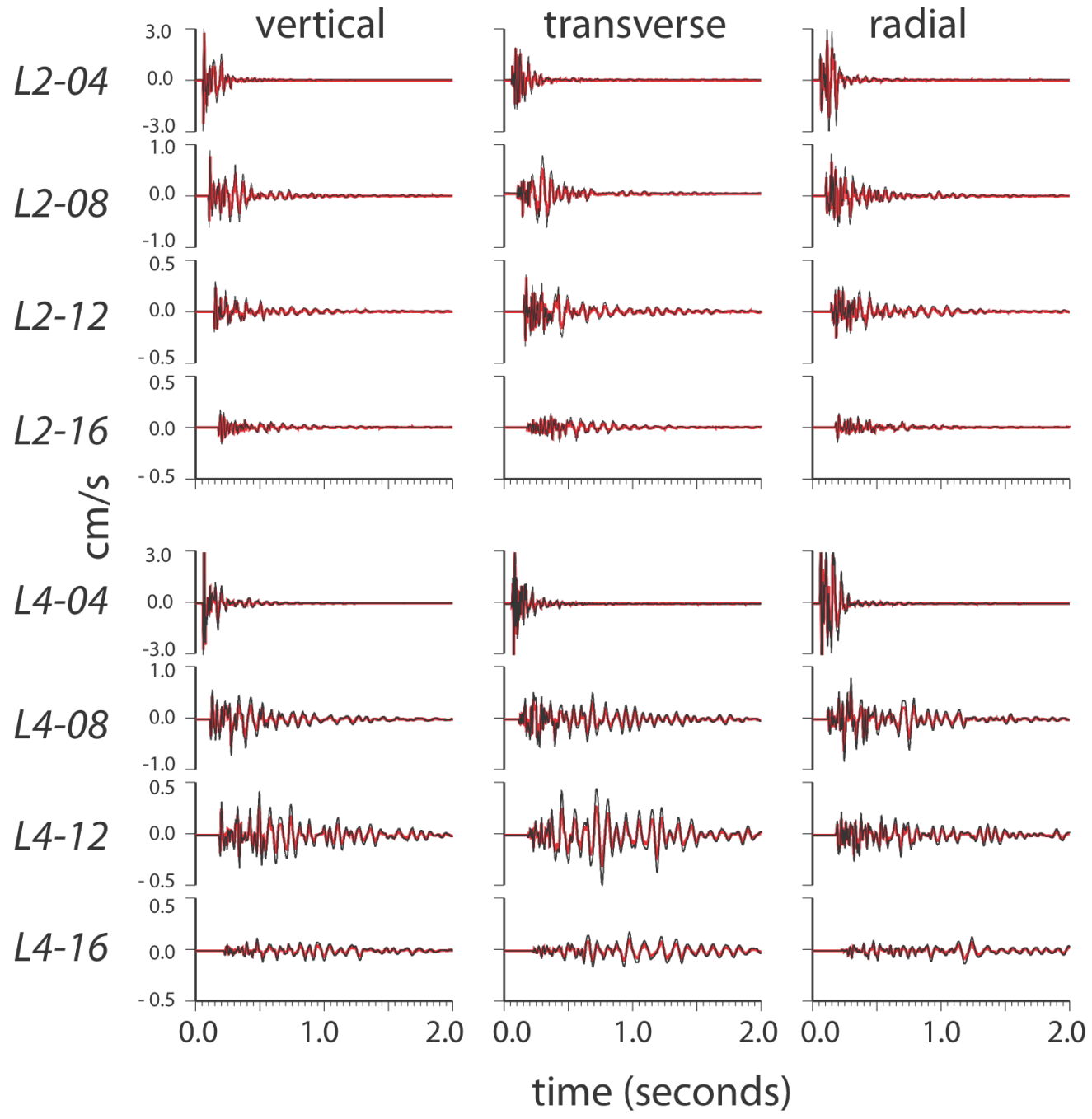

Figure 10. Seismograms for SPE2 (red) and SPE3 (black) generated by using SPE2 as a template and convolving with the expected Mueller-Murphy ratio for model A (see Figure 8). Results are similar to numerical tests with slightly higher amplitudes for SPE3. A test using model B (weaker granite) showed lower amplitudes.

amplitudes directly from the explosive source and includes coupling between the explosion and the media. Care should be taken in interpreting differences in frequency as the frequency content of an isotropic source was specified to avoid numerical effects due to grid size and wavelength; the GEODYN source terms were filtered differently during the interpolation to match the WPP node spacing. We are working on resolving these details. As each simulation requires a full GEODYN run followed by post-processing and then a full WPP simulation, each test is time-consuming. The simulations in this 
report are the first test of the 3D GEODYN/WPP coupling with a highly heterogeneous far-field model. The SPE2 GEODYN/WPP coupling only tested an axial-symmetric GEODYN model.
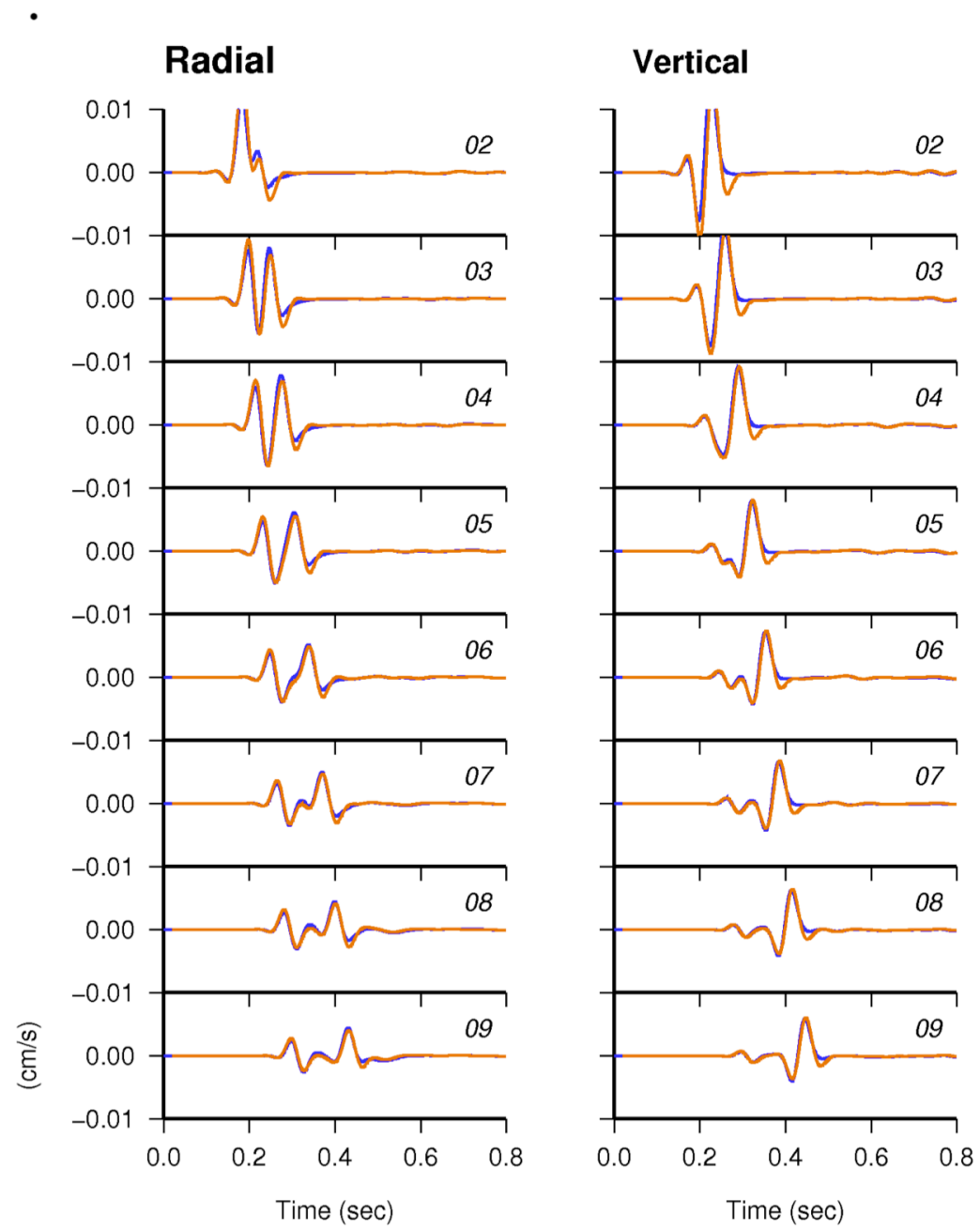

Figure 11. Comparison of synthetic velocity seismograms calculated with WPP code (brown) GEODYN-WPP codes (blue), for a half space velocity model. The seismograms are band-pass filtered at 0.1-13 Hz

Aftershock prediction. Aftershocks are one means of discriminating between underground explosions and earthquakes [Ford and Walter, 2010] and passive monitoring for aftershocks is included in the Comprehensive Nuclear-Test-Ban Treaty to refine the search area and to resolve the nature of an event [e.g. www.ctbto.org, 2011]. The existence and number of aftershocks may also depend on the nature of 
the material surrounding the explosion. Whether pre-existing damage affects the number of aftershocks has not been well addressed and therefore SPE3 provides a useful test of the idea. No clear aftershocks were recorded for SPE2, which was lower than predicted at the detection level.

\section{CONCLUSIONS}

A major challenge appears to be matching the transverse energy visible in the data, which is likely due to a combination of source and path effects. In fact, determining the partitioning of shear waves due to source and path is one of the primary goals of this experimental series. While the current synthetics generate significant transverse energy due to scattering along various lines, we have not yet included a source capable of generating transverse energy in the synthetics.

A number of improvements as well as new capabilities were added for the SPE3 predictions. The velocity model includes a higher resolution image of the granite including a weathered layer and random heterogeneities based on a statistical representation of well log measurements. Capability enhancement consists of 3D GEODYN/WPP coupling.

Future improvements:

- $\quad$ Refinement of the velocity model based on ambient noise tomography

- $\quad$ Revise model of granite with higher resolution velocity structure.

- $\quad$ GEODYN/WPP coupling with topography

- Improve visualization of the simulations for rapid assessment.

\section{Acknowledgements.}

We thank the DOE Office of Sciences, ASCR and the LDRD for support of WPP. We appreciate the efforts of NSTec personnel for efforts in conducting the SPE experiment and collecting the seismic data.

This work performed under the auspices of the U.S. Department of Energy by Lawrence Livermore National Laboratory under Contract DE-AC52-07NA27344.

\section{LLNL contribution \# LLNL-TR-XXXXXXX}

\section{REFERENCES}

Antoun, T. H., I. N. Lomov, and L. A. Glenn (2001). Development and application of a strength and damage model for rock under dynamic loading, in proceedings of the 38th U.S. Rock Mechanics Symposium, Rock Mechanics in the National Interest, D. Elsworth, J. Tinucci and K. Heasley (eds.), A. A. Balkema Publishers, Lisse, The Netherlands, 369-374. 
Antoun, T. H. and I. N. Lomov (2003). Simulation of a spherical wave experiment in marble using multidirectional damage model, 13th American Physical Society Topical Conference on Shock Compression of Condensed Matter, Portland, OR July 20-25, 2003.

Antoun, T. H., I. N. Lomov and L.A. Glenn (2004). Simulation of the penetration of a sequence of bombs into granitic rock, Int. J. Impact Eng. 29: 81-94.

Ford, S. R., and W. R. Walter (2010) Aftershock characteristics as a means of discriminating explosions from earthquakes, Bull. Seis. Soc. Amer. 100(1), 364-76, doi: 10.1785/0120080349.

Geotechnical Sciences Group, (2006), A Hydrostratigraphic Model and Alternatives for the Groundwater Flow and Contaminant Transport Model of Corrective Action Unit 97: Yucca Flat-Climax Mine, Lincoln and Nye Counties, Nevada, DOE/NV/11718-1119.

Khalturin, V. I., T. G. Rautian, and P. G. Richards, (1998), The seismic signal strength of chemical explosions, Bull. Seismo. Soc. Amer., V88, 6, 1511-1524.

Kohler, W. M. and G. S. Fuis, (1992), Empirical dependence of seismic ground velocity on the weight of explosives, shotpoint site condition, and recording distance for seismic refraction data, Bull. Seismo. Soc. Amer., 82, (5), 2032-2044.

Lomov. I. N., T. H. Antoun, J. Wagoner and J. Rambo (2003). Three-dimensional simulation of the Baneberry nuclear event, in Proceedings of the 13th American Physical Society Topical Conference on Shock Compression of Condensed Matter, Portland, OR July 20-25, 2003.

Nigbor, R. L. (1994). Six-degree-of-freedom ground motion measurement, Bull. Seismol. Soc. Am. 84, $1665-1669$.

Mellors, R. J., P. Harben, S. Ford, J. Wagoner, B. Walter, A. Rodgers, T. Hauk, S. Ruppert, S. Myers, E. Matzel, D. Dodge, M. Pasyanos, R. Gok, N. Simmons, A. Petersson, B. Sjogreen, and J. P. Lewis, (2011a), SPE 1 Data Quicklook report, LLNL-TR-485228.

Mellors, R. J., P. Harben, S. Ford, J. Wagoner, B. Walter, A. Rodgers, T. Hauk, S. Ruppert, S. Myers, E. Matzel, D. Dodge, M. Pasyanos, R. Gok, N. Simmons, A. Petersson, B. Sjogreen, and J. P. Lewis, (2011b). Analysis and simulation of far-field seismic data from the source physics experiment explosions, 2011 Monitoring Research review: Ground-Based Nuclear Explosion Monitoring Technologies, Sept. 13-15, Tuscon, AZ, LA-UR-11-04823, p. 503-511.

Mueller, R. and J. Murphy (1971). Seismic characteristics of underground nuclear detonations Part 1: Seismic source scaling, Bull. Seismol. Soc. Am. 61: 1675-1692.

Petersson, N. A. (2011). WPP software website, https://computation.llnl.gov/casc/serpentine/software.html 
Petersson, N. A. and B. Sjögreen (2009). An Energy Absorbing Far-Field Boundary Condition for the Elastic Wave Equation, Comm. Comput. Phys. 6: 483-508

Petersson, N.A., B. Sjogreen (2009), Stable grid refinement and singular source discretiztion for seismic wave simulations, Comm. Comput. Phys., v. 8, no. 5, pp. 1074-1110.

Petersson, N. A. and B. Sjögreen (2011). User's guide to WPP version 2.1, Lawrence Livermore National Laboratory technical report, LLNL-SM-487431.

Rodgers, A. J., J. Wagoner, N. A. Petersson, and B. Sjogreen, (2010), Preliminary pre-shot simulations of far-field ground motions for the source physics experiment (SPE) explosions at the Climax Stock, Nevada National Security Site, pp. 23, LLNL-TR-461990.

Rodgers, A. J., H. Xu, I. N. Lomov, N. A. Petersson, B. Sjogreen, O. Y. Vorobiev and V. Chipman, (2011) Improving ground motion simulation capabilities for underground explosion monitoring: coupling hydrodynamic-to-seismic solvers and studies of emplacement conditions, 2011 Monitoring Research review: Ground-Based Nuclear Explosion Monitoring Technologies, Sept. 13-15, Tuscon, AZ, LA-UR-11-04823, p. 363-371.

Sjögreen, B. and N. A. Petersson (2010). Stable grid refinement and singular source discretization for seismic wave simulations, Comm. Comput. Phys, LLNL-JRNL-419382.

Suryanto, W., (2006), Rotational motions in seismology, theory and application, Ph.D dissertation, pp. 118 , University of Munich. 